\title{
Twisting on associative algebras and Rota-Baxter type operators
}

\author{
Kyousuke Uchino*
}

\begin{abstract}
We introduce an operation called "twisting" on the Hochschild complex by analogy with Drinfeld's twisting operations. By using the twisting and derived bracket constructions, we study differential graded Lie algebra structures associated to the bi-graded Hochschild complex. We show that Rota-Baxter type operators are solutions of Maurer-Cartan equations. As an application of twisting, we give a construction of associative Nijenhuis operators.
\end{abstract}

Mathematics Subject Classification (2010). 13D03, 16S80, 17B62.

Keywords. Deformation theory, twisting, Rota-Baxter operators, Reynolds operators, Nijenhuis operators.

\section{Introduction}

In [7] Drinfeld introduced an operation called "twisting", motivated by the study of quasi-Lie bialgebras and quasi-Hopf algebras. The twisting operations provide a method of analyzing Manin triples. In the context of Poisson geometry, they gave a detailed study of twisting operations (see Kosmann-Schwarzbach [10], [12] and Roytenberg [21], [22]). We briefly describe twisting operations. We consider a graded commutative algebra, $\bigwedge^{*}\left(V \oplus V^{*}\right)$, where $V$ is a vector space over $\mathbb{R}$ and $V^{*}$ is the dual space of $V$. The graded algebra has a graded Poisson bracket defined by $\{V, V\}=\left\{V^{*}, V^{*}\right\}:=0$ and $\left\{V, V^{*}\right\}:=\left\langle V, V^{*}\right\rangle$. By definition, a structure in the graded Poisson algebra is an element $\Theta$ in $\bigwedge^{3}\left(V \oplus V^{*}\right)$ satisfying the Maurer-Cartan equation $\{\Theta, \Theta\}=0$. It is known that the structure $\Theta$ is an invariant Lie algebra structure on $V \oplus V^{*}$. The structures are closely related to (quasi-)Lie bialgebra structures. A Lie bialgebra structure is defined as a pair of tensors $\left(v_{1}, v_{2}\right)$ such that $\Theta_{12}:=v_{1}+v_{2}$ is a structure in the above sense, where $v_{1} \in\left(\bigwedge^{2} V^{*}\right) \otimes V$ and $v_{2} \in V^{*} \otimes \bigwedge^{2} V$. When $\left(v_{1}, v_{2}\right)$ is a structure of Lie bialgebra, the total space $\left(V \oplus V^{*}, \Theta_{12}\right)$ is called a Drinfeld double. Let $r$ be an element in $V \wedge V$. By definition, the twisting of a structure $\Theta$ by $r$ is a canonical transformation,

$$
\Theta^{r}:=\exp \left(X_{r}\right)(\Theta),
$$

\footnotetext{
* Supported by Keio University COE program.
} 
where $X_{r}$ is a Hamiltonian vector field $X_{r}:=\{-, r\}$ and $\Theta^{r}$ is the result of twisting. Interesting information is provided by the orbits of twisting operations. We recall a basic proposition. Let $\left(v_{1}, 0\right)$ be a structure of Lie bialgebra such that $v_{2}=0$. Then the Drinfeld double is the space $V \oplus V^{*}$ with the structure $\Theta_{1}:=v_{1}$. If $r$ is a solution of the Maurer-Cartan equation (or classical Yang-Baxter equation)

$$
[r, r]=0,
$$

then a pair $\left(v_{1},\left\{v_{1}, r\right\}\right)$ is a Lie bialgebra structure and the double, $v_{1}+\left\{v_{1}, r\right\}$, is equal to the result of twisting $\Theta_{1}^{r}$, where $[r, r]:=\left\{\left\{v_{1}, r\right\}, r\right\}$. Conversely, the Maurer-Cartan condition of $r$ is characterized by this property.

The aim of this note is to construct the theory of twisting on associative algebras according to the philosophy and construction in [12] and [21]. At first, we will define a twisting operation in the category of associative algebras. The twisting operation is defined by using only a canonical bigraded system of the graded Poisson algebra $\bigwedge^{*}\left(V \oplus V^{*}\right)$. Hence, given a suitable bigraded Lie algebra, one can define a twisting-like operation on the bigraded Lie algebra. We consider the Hochschild complex $C^{*}(\mathcal{T}):=\operatorname{Hom}\left(\mathcal{T}^{\otimes *}, \mathcal{T}\right)$, where $\mathcal{T}$ is a vector space decomposed into two subspaces $\mathcal{T}:=\mathcal{A}_{1} \oplus \mathcal{A}_{2}$. In Section 2 , we introduce a canonical bigraded Lie algebra system on $C^{*}\left(\mathcal{A}_{1} \oplus \mathcal{A}_{2}\right)$. The graded Lie bracket is given by Gerstenhaber's bracket product. Our structures, $\theta$, are defined as associative structures on $\mathcal{A}_{1} \oplus \mathcal{A}_{2}$, i.e., $\theta$ is a 2-cochain in $C^{2}\left(\mathcal{A}_{1} \oplus \mathcal{A}_{2}\right)$ and $t_{1} * t_{2}:=\theta\left(t_{1} \otimes t_{2}\right)$ is associative for any $t_{1}, t_{2} \in \mathcal{A}_{1} \oplus \mathcal{A}_{2}$. For a given 1-cochain $H: \mathcal{A}_{2} \rightarrow \mathcal{A}_{1}$, we define a twisting operation in the same manner as the classical one,

$$
\theta^{H}:=\exp \left(X_{\hat{H}}\right)(\theta)
$$

where $\hat{H}$ is the image of the natural map $C^{*}\left(\mathcal{A}_{2}, \mathcal{A}_{1}\right) \hookrightarrow C^{*}\left(\mathcal{A}_{1} \oplus \mathcal{A}_{2}\right), X_{\hat{H}}$ is an analogy of Hamiltonian vector field defined by $X_{\hat{H}}:=\{-, \hat{H}\}$, and where $C^{*}\left(\mathcal{A}_{2}, \mathcal{A}_{1}\right):=\operatorname{Hom}\left(\mathcal{A}_{2}^{\otimes *}, \mathcal{A}_{1}\right)$. We will see that $\theta$ is decomposed into the unique four substructures

$$
\theta=\hat{\phi}_{1}+\hat{\mu}_{1}+\hat{\mu}_{2}+\hat{\phi}_{2} .
$$

The twisting operation is completely determined by transformation rules of the four substructures. In Section 4 we present explicit formulas of the transformation rules (Theorem 4.5).

We consider the case of $\hat{\phi}_{1}=\hat{\phi}_{2}=0$. Then $\mathcal{A}_{1}$ and $\mathcal{A}_{2}$ are both subalgebras of the associative algebra $\left(\mathcal{A}_{1} \oplus \mathcal{A}_{2}, \theta\right)$. Such a triple $\left(\mathcal{A}_{1} \oplus \mathcal{A}_{2}, \mathcal{A}_{1}, \mathcal{A}_{2}\right)$ is called associative twilled algebra, simply, twilled algebra (Carinena et al. [5]). If a Lie algebra decomposes into two subalgebras, it is called a twilled Lie algebra in [11], or called a twilled extension in [13], or a double Lie algebra in [17]. This concept is used in order to construct integrable Hamiltonian systems (Adler-Kostant-Symes theorem). The notion of associative twilled algebra considered here is an associative version of the classical one. In [5] associative twilled algebras are studied from the 
point of view of quantization. In Section 3 we will perform a detailed investigation of twilled algebras. By the derived bracket construction in [11], a twilled algebra structure on $\mathcal{A}_{1} \oplus \mathcal{A}_{2}$ induces a differential graded Lie algebra (briefly dg-Lie algebra) structure on $C^{*}\left(\mathcal{A}_{2}, \mathcal{A}_{1}\right)$ (see Proposition 3.3). So we can consider a deformation theory on the induced dg-Lie algebra. We consider the Maurer-Cartan equation

$$
d R+\frac{1}{2}[R, R]=0
$$

in a dg-Lie algebra and find a solution $R$ in a Rota-Baxter algebra. Let $(\mathcal{A}, R)$ be an arbitrary associative algebra equipped with an operator $R: \mathcal{A} \rightarrow \mathcal{A}$. The operator $R$ is called Rota-Baxter operator if $R$ satisfies the identity (so-called Rota-Baxter identity)

$$
R(x) R(y)=R(R(x) y+x R(y))+q R(x y),
$$

where $q \in \mathbb{K}$ is a scalar (called weight). Rota-Baxter operators have been studied in combinatorics (see Rota [18], [19]). In this note we do not pursue this line because it is beyond our aim. Now $\mathcal{A} \oplus \mathcal{A}$ has a natural twilled algebra structure, and then $C^{*}(\mathcal{A}, \mathcal{A})$ has a dg-Lie algebra structure. In Section 5.1, we show that $R$ is a RotaBaxter operator if and only if $R$ is a solution of the Maurer-Cartan equation.

In Section 6 we give an application of our construction. We recall the notion of associative Nijenhuis operator ([5]). Let $N: \mathcal{A} \rightarrow \mathcal{A}$ be a linear map on an associative algebra $\mathcal{A}$. The operator $N$ is called associative Nijenhuis operator if it satisfies an associative version of the classical Nijenhuis condition

$$
N(x) N(y)=N(N(x) y+x N(y))-N^{2}(x y),
$$

where $x, y \in \mathcal{A}$. The deformed multiplication $x \times_{N} y:=N(x) y+x N(y)-N(x y)$ is a new associative multiplication on $\mathcal{A}$ which is compatible with the original one. In this sense, an associative Nijenhuis operator induces a quantum bihamiltonian system (see [5]). We give a construction of associative Nijenhuis operators by analogy with Poisson-Nijenhuis geometry.

We recall a theorem of Vaisman [25]. Let $(V, P)$ be a Poisson manifold equipped with a Poisson structure tensor $P$, i.e., $P$ is a solution of the Maurer-Cartan equation

$$
\frac{1}{2}[P, P]=0,
$$

where the bracket product is a graded Lie bracket (called Schouten-Nijenhuis bracket). Since the Poisson structure is a $(2,0)$-tensor, it is identified with a bundle map $P: T^{*} V \rightarrow T V$. The Poisson bundle map induces a Lie algebroid structure on the cotangent bundle $T^{*} V$, i.e., the space of sections of $\wedge^{*} T^{*} V$ has a certain graded Lie bracket $\{,\}_{P}$. Vaisman showed that if a 2-form $\omega$ is a solution of the strong Maurer-Cartan equation $d \omega=\{\omega, \omega\}_{P}=0$, then the bundle map $N:=P \omega: T V \rightarrow T V$ is a Nijenhuis tensor and the pair $(P, N)$ is a compatible pair or a Poisson-Nijenhuis structure in the sense of [14]. This compatibility implies that 
the bundle map $N P: T^{*} V \rightarrow T V$ is a Poisson structure bundle map and $P+t N P$ is a one-parameter family of Poisson structures.

We will obtain a result similar to Vaisman's theorem. First of all, we need RotaBaxter type operators as substitutes for Poisson structures. Let $\mathcal{A}$ be an associative algebra and $M$ an $\mathcal{A}$-bimodule, and let $\pi: M \rightarrow \mathcal{A}$ be a linear map. The linear map $\pi$ is called a generalized Rota-Baxter operator of weight 0 , or briefly GRB ([23]), if $\pi$ is a solution of

$$
\pi(m) \pi(n)=\pi(\pi(m) \cdot n+m \cdot \pi(n)),
$$

where $m, n \in M$ and - is the bimodule action. When $M=\mathcal{A}$ as a canonical bimodule, (GRB) reduces to a classical Rota-Baxter identity of weight zero. We consider a semi-direct product algebra $\mathcal{T}:=(A \ltimes M, \hat{\mu})$, where $\hat{\mu}$ is the associative multiplication of $\mathcal{A} \ltimes M$. The Hochschild complex $C^{*}(\mathcal{A} \ltimes M)$ becomes a dg-Lie algebra by Gerstenhaber bracket and the coboundary map $d_{\hat{\mu}}:=\{\hat{\mu},-\}$. We define, due to [11], a second bracket product on $C^{*}(\mathcal{A} \ltimes M)$ by

$$
[f, g]_{\hat{\mu}}:=(-1)^{|f|-1}\{\{\hat{\mu}, f\}, g\} .
$$

Here the new bracket is a graded Lie bracket on $C^{*}(M, \mathcal{A}) \subset C^{*}(\mathcal{A} \ltimes M)$. One can show that $\pi$ is a generalized Rota-Baxter operator if and only if it is a solution of the Maurer-Cartan equation

$$
\frac{1}{2}[\hat{\pi}, \hat{\pi}]_{\hat{\mu}}=0,
$$

where $\hat{\pi}$ is the image of the natural map $C^{1}(M, \mathcal{A}) \hookrightarrow C^{1}(\mathcal{A} \ltimes M), \pi \mapsto \hat{\pi}$.

Now, given a generalized Rota-Baxter operator $\pi: M \rightarrow \mathcal{A}, M$ becomes an associative algebra, where the associative multiplication on $M$ is given by a structure $\{\hat{\mu}, \hat{\pi}\}$. The associativity of $\{\hat{\mu}, \hat{\pi}\}$ follows from $[\hat{\pi}, \hat{\pi}]_{\hat{\mu}}=0$. We denote the associative algebra by $M_{\pi}$. One can show that $M_{\pi} \oplus \mathcal{A}$ has a twilled algebra structure. Thus a dg-Lie algebra structure, $\left(d_{\hat{\mu}},[,]_{\{\hat{\mu}, \hat{\pi}\}}\right)$, is induced on $C^{*}\left(\mathcal{A}, M_{\pi}\right)$. By analogy with Vaisman's theorem, we assume that $\Omega: \mathcal{A} \rightarrow M$ is a solution of the strong Maurer-Cartan equation in $C^{*}\left(\mathcal{A}, M_{\pi}\right)$,

$$
d_{\hat{\mu}} \widehat{\Omega}=[\hat{\Omega}, \widehat{\Omega}]_{\{\hat{\mu}, \hat{\pi}\}}=0,
$$

where $d_{\hat{\mu}}$ is the Hochschild coboundary on $C^{*}(\mathcal{A}, M)$ and $\widehat{\Omega}$ is defined similarly with $\hat{\pi}$. Then we can show that a linear endomorphism $N:=\pi \Omega: \mathcal{A} \rightarrow \mathcal{A}$ is an associative Nijenhuis operator and the pair $(\pi, N=\pi \Omega)$ is compatible (see Proposition 6.1). This proposition can be considered as an associative version of Vaisman's result.

Acknowledgements. The author wishes to thank very much the referees. He is greatly indebted to them for their numerous suggestions. Finally, he would like to thank very much Professors Jean-Louis Loday, Yoshiaki Maeda and Akira Yoshioka for helpful comments and encouragement. 


\section{Cochain calculus}

In this section, we define a bigraded Lie algebra structure on Hochschild complex $C^{*}\left(\mathcal{A}_{1} \oplus \mathcal{A}_{2}\right)$. In the following, we assume that the characteristic of the ground field $\mathbb{K}$ is zero and so $\mathbb{Q}$ is included in $\mathbb{K}$.

2.1. Gerstenhaber brackets. We recall the definition of Gerstenhaber's bracket product. Let $V$ be a vector space over $\mathbb{K}$. We consider the space of cochains $\mathfrak{g}(V):=$ $\bigoplus_{n \in \mathbb{N}} C^{n}(V)$, where $C^{n}(V)=C^{n}(V, V):=\operatorname{Hom}_{\mathbb{K}}\left(V^{\otimes n}, V\right)$. The degree of $f \in g(V)$ is denoted by $|f|$, so $f$ is in $C^{|f|}(V)$. For any $f \in C^{|f|}(V)$ and $g \in C^{|g|}(V)$, we define a product,

$$
f \bar{\circ} g:=\sum_{i=1}^{|f|}(-1)^{(i-1)(|g|-1)} f \circ_{i} g,
$$

where $\circ_{i}$ is the composition of maps defined by

$$
\begin{aligned}
& f \circ_{i} g\left(b_{1}, \ldots, b_{|f|+|g|-1}\right) \\
& \quad=f\left(b_{1}, \ldots, b_{i-1}, g\left(b_{i}, \ldots, b_{i+|g|-1}\right), b_{i+|g|} \ldots, b_{|f|+|g|-1}\right) .
\end{aligned}
$$

The degree of $f \bar{\circ} g$ is $|f|+|g|-1$. The Gerstenhaber bracket, or shortly, G-bracket on $\mathrm{g}(V)$ is defined as a graded commutator,

$$
\{f, g\}:=f \bar{\circ} g-(-1)^{(|f|-1)(|g|-1)} g \bar{\circ} f .
$$

We recall two fundamental identities.

(I) Graded commutativity:

$$
\{f, g\}=-(-1)^{(|f|-1)(|g|-1)}\{g, f\} ;
$$

(II) graded Jacobi identity:

$$
\begin{aligned}
(-1)^{(|f|-1)(|h|-1)}\{\{f, g\}, h\} & +(-1)^{(|h|-1)(|g|-1)}\{\{h, f\}, g\} \\
& +(-1)^{(|g|-1)(|f|-1)}\{\{g, h\}, f\}=0,
\end{aligned}
$$

where $h \in C^{|h|}(V)$. The above graded Jacobi identity is equivalent to

$$
\{f,\{g, h\}\}=\{\{f, g\}, h\}+(-1)^{(|f|-1)(|g|-1)}\{g,\{f, h\}\},
$$

which is called graded Leibniz identity, sometimes also called graded Loday identity.

Graded Lie algebras. Let $\mathrm{g}$ be a graded vector space equipped with a binary multiplication $\{$,$\} of degree 0$. If the bracket product satisfies the two conditions

$$
\begin{aligned}
\{f, g\} & =-(-1)^{\operatorname{deg}(f) \operatorname{deg}(g)}\{g, f\}, \\
\{f,\{g, h\}\} & =\{\{f, g\}, h\}+(-1)^{\operatorname{deg}(f) \operatorname{deg}(g)}\{g,\{f, h\}\},
\end{aligned}
$$


then $\mathfrak{g}$ is called a graded Lie algebra. Here $f, g, h, \in \mathfrak{g}$ and $\operatorname{deg}(-)$ is the degree of $\mathfrak{g}$. The cochain complex $g(V)$ is a graded Lie algebra of $\operatorname{deg}(f):=|f|-1$. A graded Lie algebra $\mathfrak{g}$ is called a differential graded Lie algebra (dg-Lie algebra) if $\mathfrak{g}$ has a square zero derivation $d$ of degree +1 satisfying

$$
d\{f, g\}=\{d f, g\}+(-1)^{\operatorname{deg}(f)}\{f, d g\} .
$$

Associative structures. It is well known that $S \in C^{2}(V)$ is an associative structure if and only if it is a solution of Maurer-Cartan equation $\{S, S\}=0$. If $S$ is an associative structure, then $d_{S}(f):=\{S, f\}$ is a coboundary map of the Hochschild complex $\left(C^{*}(V), d_{S}\right)$ so that $\left(\mathrm{g}(V), d_{S}\right)$ becomes a dg-Lie algebra.

Derived brackets. Let $g$ be a dg-Lie algebra. We define a new bracket product by

$$
[f, g]_{d}:=(-1)^{\operatorname{deg}(f)}\{d f, g\} .
$$

The new bracket is called derived bracket ([11]). It is well known that the derived bracket is a graded Leibniz bracket, i.e., (2) holds up to degree shift. Remark that the derived bracket is not graded commutative in general. We recall a basic lemma.

Lemma 2.1 ([11]). Let $\mathrm{g}$ be a dg-Lie algebra, and let $\mathfrak{h} \subset \mathrm{g}$ be an abelian subalgebra, i.e., $\{\mathfrak{h}, \mathfrak{h}\}=0$. We define a new degree (derived degree) by $\operatorname{deg}_{d}(h):=\operatorname{deg}(h)+1$. If the derived bracket is closed on $\mathfrak{h}$, then $\left(\mathfrak{h}, \operatorname{deg}_{d},[,]_{d}\right)$ is a graded Lie algebra.

2.2. Lift and bidegree. Let $\mathcal{A}_{1}$ and $\mathcal{A}_{2}$ be vector spaces, and let $c: \mathcal{A}_{2}^{\otimes n} \rightarrow \mathcal{A}_{1}$ be a linear map, or a cochain in $C^{n}\left(\mathcal{A}_{2}, \mathcal{A}_{1}\right)$. We can construct a cochain $\hat{c} \in$ $C^{n}\left(\mathcal{A}_{1} \oplus \mathcal{A}_{2}\right)$ by

$$
\hat{c}\left(\left(a_{1}, x_{1}\right) \otimes \cdots \otimes\left(a_{n}, x_{n}\right)\right):=\left(c\left(x_{1}, \ldots, x_{n}\right), 0\right) .
$$

In general, for a given multilinear map $f: \mathcal{A}_{i(1)} \otimes \mathcal{A}_{i(2)} \otimes \cdots \otimes \mathcal{A}_{i(n)} \rightarrow \mathcal{A}_{j}$, $i(1), \ldots, i(n), j \in\{1,2\}$, we define a cochain $\hat{f} \in C^{n}\left(\mathcal{A}_{1} \oplus \mathcal{A}_{2}\right)$ by

$$
\hat{f}:= \begin{cases}f & \text { on } \mathcal{A}_{i(1)} \otimes \mathcal{A}_{i(2)} \otimes \cdots \otimes \mathcal{A}_{i(n)}, \\ 0 & \text { all other cases. }\end{cases}
$$

We call the cochain $\hat{f}$ a horizontal lift of $f$, or simply lift. For instance, the lifts of $\alpha: \mathcal{A}_{1} \otimes \mathcal{A}_{1} \rightarrow \mathcal{A}_{1}, \beta: \mathcal{A}_{1} \otimes \mathcal{A}_{2} \rightarrow \mathcal{A}_{2}$ and $\gamma: \mathcal{A}_{2} \otimes \mathcal{A}_{1} \rightarrow \mathcal{A}_{2}$ are defined by

$$
\begin{aligned}
& \hat{\alpha}((a, x),(b, y))=(\alpha(a, b), 0), \\
& \hat{\beta}((a, x),(b, y))=(0, \beta(a, y)), \\
& \hat{\gamma}((a, x),(b, y))=(0, \gamma(x, b)),
\end{aligned}
$$


respectively. Let $H: \mathcal{A}_{2} \rightarrow \mathcal{A}_{1}$ (resp. $H: \mathcal{A}_{1} \rightarrow \mathcal{A}_{2}$ ) be a 1-cochain. The lift is defined by

$$
\hat{H}(a, x)=(H(x), 0) \quad(\text { resp. } \hat{H}(a, x)=(0, H(a))) .
$$

For any $(a, x) \in \mathcal{A}_{1} \oplus \mathcal{A}_{2}$, we have $\hat{H} \hat{H}(a, x)=\hat{H}(H(x), 0)=(0,0)$.

Lemma 2.2. $\hat{H} \hat{H}=0$.

This lemma will be used in Section 4.

We denote by $\mathcal{A}^{l, k}$ the direct sum of all $(l+k)$-tensor powers of $\mathcal{A}_{1}$ and $\mathcal{A}_{2}$, where $l$ (resp. $k$ ) is the number of $\mathcal{A}_{1}$ (resp. $\mathcal{A}_{2}$ ). For instance,

$$
\mathcal{A}^{1,2}:=\left(\mathcal{A}_{1} \otimes \mathcal{A}_{2} \otimes \mathcal{A}_{2}\right) \oplus\left(\mathcal{A}_{2} \otimes \mathcal{A}_{1} \otimes \mathcal{A}_{2}\right) \oplus\left(\mathcal{A}_{2} \otimes \mathcal{A}_{2} \otimes \mathcal{A}_{1}\right) .
$$

The tensor space $\left(\mathscr{A}_{1} \oplus \mathcal{A}_{2}\right)^{\otimes n}$ is expanded into the direct sum of $\mathcal{A}^{l, k}, l+k=n$. For instance,

$$
\left(\mathcal{A}_{1} \oplus \mathcal{A}_{2}\right)^{\otimes 2}=\mathcal{A}^{2,0} \oplus \mathcal{A}^{1,1} \oplus \mathcal{A}^{0,2} .
$$

We consider the space of cochains $C^{n}\left(\mathcal{A}_{1} \oplus \mathcal{A}_{2}\right):=\operatorname{Hom}_{\mathbb{K}}\left(\left(\mathcal{A}_{1} \oplus \mathcal{A}_{2}\right)^{\otimes n}, \mathcal{A}_{1} \oplus \mathcal{A}_{2}\right)$. By the standard properties of Hom-functor, we have

$$
C^{n}\left(\mathcal{A}_{1} \oplus \mathcal{A}_{2}\right) \cong \sum_{l+k=n} C^{n}\left(\mathcal{A}^{l, k}, \mathcal{A}_{1}\right) \oplus \sum_{l+k=n} C^{n}\left(\mathcal{A}^{l, k}, \mathcal{A}_{2}\right),
$$

where the isomorphism is the horizontal lift.

Let $f$ be an $n$-cochain in $C^{n}\left(\mathcal{A}_{1} \oplus \mathcal{A}_{2}\right)$. We say that the bidegree of $f$ is $k \mid l$ if $f$ is an element in $C^{n}\left(\mathscr{A}^{l, k-1}, \mathcal{A}_{1}\right)$ or in $C^{n}\left(\mathcal{A}^{l-1, k}, \mathcal{A}_{2}\right)$, where $n=l+k-1$. We denote the bidegree of $f$ by $\|f\|=k \mid l$. In general, cochains do not have bidegree. We call a cochain $f$ a homogeneous cochain if $f$ has a bidegree.

We have $k+l \geq 2$ because $n \geq 1$. Thus there are no cochains of bidegree $0 \mid 0$ or $1 \mid 0$ or $0 \mid 1$. If the dimension of $\mathcal{A}_{1}$ is finite and if $\mathcal{A}_{2}=\mathcal{A}_{1}^{*}$ is the dual space of $\mathcal{A}_{1}$, then a $k \mid l$-cochain is identified with an element in $\mathcal{A}_{1}^{\otimes k} \otimes \mathcal{A}_{1}^{* \otimes l}$. Hence the definition above is compatible with the classical one. For instance, the lift $\hat{H} \in C^{1}\left(\mathcal{A}_{1} \oplus \mathcal{A}_{2}\right)$ of $H: \mathcal{A}_{2} \rightarrow \mathcal{A}_{1}$ has bidegree $2 \mid 0$. We recall that $\hat{\alpha}, \hat{\beta}, \hat{\gamma} \in C^{2}\left(\mathcal{A}_{1} \oplus \mathcal{A}_{2}\right)$ in (4), (5) and (6). One can easily see that $\|\hat{\alpha}\|=\|\hat{\beta}\|=\|\hat{\gamma}\|=1 \mid 2$. Thus the sum

$$
\hat{\mu}:=\hat{\alpha}+\hat{\beta}+\hat{\gamma}
$$

is a homogeneous cochain of bidegree $1 \mid 2$. The cochain $\hat{\mu}$ is a multiplication of semidirect product type,

$$
\hat{\mu}((a, x),(b, y))=(\alpha(a, b), \beta(a, y)+\gamma(x, b)),
$$

where $(a, x),(b, y) \in \mathcal{T}$. Observe that $\hat{\mu}$ is not a lift (there is no $\mu$ ), however, we will use this symbol because $\hat{\mu}$ is an interesting homogeneous cochain.

Clearly, the following lemma holds. 
Lemma 2.3. Let $f \in C^{n}\left(\mathcal{A}_{1} \oplus \mathcal{A}_{2}\right)$ be a cochain. The bidegree of $f$ is $k \mid l$ if and only if the following four conditions hold:

(deg1) $k+l-1=n$.

(deg2-1) If $\boldsymbol{x}$ is an element in $\mathcal{A}^{l, k-1}$, then $f(\boldsymbol{x})$ is in $\mathcal{A}_{1}$.

(deg2-2) If $\boldsymbol{x}$ is an element in $\mathcal{A}^{l-1, k}$, then $f(\boldsymbol{x})$ is in $\mathcal{A}_{2}$.

(deg3) All the other cases, $f(x)=0$.

Lemma 2.4. If $\|f\|=k \mid 0$ (resp. $0 \mid k$ ) and $\|g\|=l \mid 0$ (resp. $0 \mid l$ ), then $\{f, g\}=0$, or simply,

$$
\{(k \mid 0),(l \mid 0)\}=\{(0 \mid k),(0 \mid l)\}=0 .
$$

Proof. Assume that $\|f\|=k \mid 0$ and $\|g\|=l \mid 0$. Then $f$ and $g$ are both horizontal lifts of cochains in $C^{*}\left(\mathcal{A}_{2}, \mathcal{A}_{1}\right)$. Thus, from the definition of lift, we have $f \circ_{i} g=$ $g \circ_{j} f=0$ for any $i, j$.

Lemma 2.5. Let $f \in C^{|f|}\left(\mathcal{A}_{1} \oplus \mathcal{A}_{2}\right)$ and $g \in C^{|g|}\left(\mathcal{A}_{1} \oplus \mathcal{A}_{2}\right)$ be homogeneous cochains with bidegrees $k_{f} \mid l_{f}$ and $k_{g} \mid l_{g}$ respectively, where $|f|$ and $|g|$ are usual degrees of cochains $f$ and $g$. The composition $f \circ_{i} g$ is again a homogeneous cochain, and the bidegree is $k_{f}+k_{g}-1 \mid l_{f}+l_{g}-1$.

Proof. We show that conditions (deg1)-(deg3) hold. The condition (deg1) holds because $k_{f}+k_{g}-1+l_{f}+l_{g}-1=|f|+|g|=\left|f \circ_{i} g\right|+1$. We show that condition (deg2) holds. Take an element $\boldsymbol{x} \otimes \boldsymbol{y} \otimes \boldsymbol{z}$ in $\mathcal{A}^{l_{f}+l_{g}-1, k_{f}+k_{g}-2}$. Consider

$$
f \circ_{i} g(\boldsymbol{x}, \boldsymbol{y}, z)=f(\boldsymbol{x}, g(\boldsymbol{y}), z) .
$$

If ( $\star$ ) is zero, then it is in $\mathcal{A}_{1}$ for (deg2-1) is satisfied. So we assume $(\star) \neq 0$. We consider the case of $g(\boldsymbol{y}) \in \mathcal{A}_{1}$. In this case, $\boldsymbol{y}$ is in $\mathcal{A}^{l_{g}, k_{g}-1}$ and $\boldsymbol{x} \otimes \boldsymbol{z}$ is in $\mathcal{A}^{l_{f}-1, k_{f}-1}$. Thus $\boldsymbol{x} \otimes g(\boldsymbol{y}) \otimes \boldsymbol{z}$ is an element in $\mathcal{A}^{l_{f}, k_{f}-1}$, which implies $f(x \otimes g(y) \otimes z) \in \mathcal{A}_{1}$. If $g(\boldsymbol{y}) \in \mathcal{A}_{2}, \boldsymbol{y}$ is in $\mathcal{A}^{l_{g}-1, k_{g}}$ and $\boldsymbol{x} \otimes z$ is in $\mathcal{A}^{l_{f}, k_{f}-2}$. Thus $\boldsymbol{x} \otimes g(\boldsymbol{y}) \otimes \boldsymbol{z}$ is an element in $\mathcal{A}^{l_{f}, k_{f}-1}$, which gives $f(\boldsymbol{x} \otimes g(\boldsymbol{y}) \otimes \boldsymbol{z}) \in \mathcal{A}_{1}$. Similarly, if $\boldsymbol{x} \otimes \boldsymbol{y} \otimes \boldsymbol{z}$ is an element in $\mathcal{A}^{l_{f}+l_{g}-2, k_{f}+k_{g}-1}$, the condition holds. We show (deg3). If $\boldsymbol{x} \otimes \boldsymbol{y} \otimes \boldsymbol{z}$ is an element in $\mathcal{A}^{l_{f}+l_{g}-1+i, k_{f}+k_{g}-2-i}$ and $g(\boldsymbol{y}) \neq 0$, then $\boldsymbol{x} \otimes g(\boldsymbol{y}) \otimes \boldsymbol{z}$ is in $\mathcal{A}^{l_{f}+i, k_{f}-1-i}$. When $i \neq 0$, from the assumption, $f(\boldsymbol{x} \otimes g(\boldsymbol{y}) \otimes z)=0$. The proof is completed.

Proposition 2.6. If $\|f\|=k_{f} \mid l_{f}$ and $\|g\|=k_{g} \mid l_{g}$, then the Gerstenhaber bracket $\{f, g\}$ has the bidegree $k_{f}+k_{g}-1 \mid l_{f}+l_{g}-1$.

Proof. Straightforward.

Remark. Given a bidegree $k+1 \mid l+1$-cochain $f$, we define $\operatorname{bideg}(f):=k \mid l$. If $\operatorname{bideg}(f)=k \mid l$ and $\operatorname{bideg}(g)=m \mid n$, then $\operatorname{bideg}(\{f, g\})=\operatorname{bideg}(f)+\operatorname{bideg}(g)=$ $k+m \mid l+n$. Thus the bidegree, bideg, of the Gerstenhaber bracket is $0 \mid 0$. 


\section{Main objects}

Notation. Let $\mathcal{A}_{1}$ and $\mathscr{A}_{2}$ be two vector spaces. We denote the elements of $\mathcal{A}_{1}$ by $a, b, c, \ldots$ and the elements of $\mathcal{A}_{2}$ by $x, y, z, \ldots$ We sometimes use an identification $(a, x) \cong a+x$, where $(a, x) \in \mathcal{A}_{1} \oplus \mathcal{A}_{2}$.

\subsection{Twilled algebras}

3.1.1. Structures. Let $\mathcal{T}$ be an associative algebra equipped with an associative product $\theta$. We assume a decomposition of $\mathcal{T}$ into two subspaces, $\mathcal{T}=\mathcal{A}_{1} \oplus \mathcal{A}_{2}$. The associative structure defines an associative multiplication by $\theta((a, x),(b, y)):=$ $(a, x) *(b, y)$ for any $(a, x),(b, y) \in \mathcal{T}$.

Definition 3.1 ([5]). The triple $\left(\mathcal{T}, \mathcal{A}_{1}, \mathcal{A}_{2}\right)$, or simply $\mathcal{T}$, is called an associative twilled algebra if $\mathcal{A}_{1}$ and $\mathcal{A}_{2}$ are subalgebras of $\mathcal{T}$. We sometimes denote a twilled algebra $\mathcal{T}$ by $\mathcal{A}_{1} \bowtie \mathcal{A}_{2}$. Such a structure is sometimes called a matching pair of associative algebras in the literature.

One can easily check that if $\mathcal{A}_{1} \bowtie \mathcal{A}_{2}$ is a twilled algebra, then $\mathcal{A}_{1}$ (resp. $\mathcal{A}_{2}$ ) is an $\mathcal{A}_{2}$-bimodule (resp. $\mathcal{A}_{1}$-bimodule). These bimodule structures are defined by the following decomposition of associative multiplication of $\mathcal{T}$. For any $a \in \mathcal{A}_{1}$ and $x \in \mathcal{A}_{2}$, the multiplications $a * x$ and $x * a$ are decomposed into four multiplications,

$$
a * x=\left(a *_{2} x, a *_{1} x\right), \quad x * a=\left(x *_{2} a, x *_{1} a\right),
$$

where $a *_{2} x$ and $x *_{2} a$ are $\mathcal{A}_{1}$-components of $a * x$ and $x * a$, respectively, and, similarly, $a *_{1} x$ and $x *_{1} a$ are $\mathcal{A}_{2}$-components. One can easily check that the multiplication $*_{1}\left(\right.$ resp. $\left.*_{2}\right)$ is the bimodule action of $\mathcal{A}_{1}$ on $\mathcal{A}_{2}$ (resp. $\mathcal{A}_{2}$ on $\left.\mathcal{A}_{1}\right)$.

In general, the associative multiplication of $\mathcal{A}_{1} \bowtie \mathcal{A}_{2}$ has the form

$$
(a, x) *(b, y)=\left(a * b+a *_{2} y+x *_{2} b, a *_{1} y+x *_{1} b+x * y\right) .
$$

The total multiplication, $*$, is decomposed into two "associative" multiplications of semidirect product,

$$
\begin{aligned}
& (a, x) *_{1}(b, y):=\left(a *_{1} b, a *_{1} y+x *_{1} b\right), \\
& (a, x) *_{2}(b, y):=\left(a *_{2} y+x *_{2} b, x *_{2} y\right),
\end{aligned}
$$

where we put $a *_{1} b:=a * b$ and $x *_{2} y:=x * y$. Hence the structure $\theta$ is also decomposed into two associative structures,

$$
\theta=\hat{\mu}_{1}+\hat{\mu}_{2},
$$

where $\hat{\mu}_{i}$ is the structure associated with the multiplication $*_{i}$ for $i=1,2$. Recall (8). The cochains $\hat{\mu}_{1}$ and $\hat{\mu}_{2}$ have the bidegrees $1 \mid 2$ and $2 \mid 1$, respectively. Under the assumption, the decomposition of $\theta$ is unique, i.e., if $\theta$ is decomposed into two substructures of bidegrees $1 \mid 2$ and $2 \mid 1$, then such substructures are uniquely determined. 
Lemma 3.2. The associativity of $\theta(\{\theta, \theta\}=0)$ is equivalent to the compatibility conditions

$$
\begin{aligned}
\frac{1}{2}\left\{\hat{\mu}_{1}, \hat{\mu}_{1}\right\} & =0, \\
\left\{\hat{\mu}_{1}, \hat{\mu}_{2}\right\} & =0, \\
\frac{1}{2}\left\{\hat{\mu}_{2}, \hat{\mu}_{2}\right\} & =0 .
\end{aligned}
$$

Proof. We will show a more general result in Lemma 3.9 below.

3.1.2. The case of subalgebras in duality. Given an arbitrary associative algebra $\mathcal{A}$, we have a Lie algebra by the commutator $[a, b]:=a b-b a$ on $\mathcal{A}$. The induced Lie algebra is denoted by $L(\mathcal{A})$. The correspondence $L: \mathcal{A} \rightarrow L(\mathcal{A})$ is a functor (sometimes called "Liezation") from the usual category of associative algebras to the category of Lie algebras.

In this short section, we assume that $\mathcal{A}_{1}=: \mathcal{A}$ is a finite dimensional vector space and $\mathcal{A}_{2}$ is the dual space. In this case, $\mathcal{T}=\mathcal{A} \oplus \mathcal{A}^{*}$ has a nondegenerate symmetric bilinear form, $(-\mid-)$, where $\left(\mathcal{A} \mid \mathcal{A}^{*}\right)=\left(\mathcal{A}^{*} \mid \mathcal{A}\right)$ is the dual pairing and $(\mathscr{A} \mid \mathcal{A})=\left(\mathcal{A}^{*} \mid \mathcal{A}^{*}\right)=0$. We make a natural assumption, namely, the bilinear form is invariant (or associative) with respect to the associative multiplication of $\mathcal{J}$, explicitly,

$$
\left(t_{1} * t_{2} \mid t_{3}\right)=\left(t_{1} \mid t_{2} * t_{3}\right)
$$

for any $t_{1}, t_{2}, t_{3} \in \mathcal{T}$. Such a twilled algebra is called invariant twilled algebra.

If $\mathcal{T}$ is an invariant twilled algebra, then the triple $\left(L(\mathcal{T}), L(\mathcal{A}), L\left(\mathcal{A}^{*}\right)\right)$ is a Manin triple. It is a twilled Lie algebra with an invariant pseudo-Euclidean metric decomposed into two maximally isotropic subalgebras. In general, a pair of Lie algebras $\left(g_{1}, g_{2}\right)$ becomes a Lie bialgebra if and only if a triple of Lie algebras $\left(\mathfrak{g}_{1} \bowtie \mathfrak{g}_{2}, \mathfrak{g}_{1}, \mathfrak{g}_{2}\right)$ is a Manin triple. In this case, the total space $\mathfrak{g}_{1} \bowtie \mathfrak{g}_{2}$ is called a Drinfeld double. Thus the pair $\left(L(\mathcal{A}), L\left(\mathcal{A}^{*}\right)\right)$ becomes a Lie bialgebra and $L(\mathcal{A}) \bowtie L\left(\mathcal{A}^{*}\right)$ is a Drinfeld double. If $\mathcal{T}$ is a quasi-twilled algebra in the sense of Definition 3.10 below, then the cocycle term $\phi_{1}\left(\right.$ or $\left.\phi_{2}\right)$ is a cyclic cocycle, i.e., for any $a, b, c \in \mathcal{A}$,

$$
\phi_{1}(a, b)(c)=\phi_{1}(b, c)(a)=\phi_{1}(c, a)(b) .
$$

This fact is a direct consequence of the invariance property. The commutator $\Phi_{1}(a, b):=\phi_{1}(a, b)-\phi_{1}(b, a)$ is identified with a skew-symmetric 3-tensor in $\bigwedge^{3} \mathcal{A}^{*}$. This implies that if $\mathscr{A} \oplus \mathcal{A}^{*}$ is a quasi-twilled algebra, then $L(\mathcal{T})$ is the double of quasi-Lie bialgebra $\left(L(\mathcal{A}), L(\mathcal{A})^{*}\right)$ (see [7], [10] for quasi-Lie bialgebras).

The dual map of an associative multiplication on $\mathcal{T}$ becomes a coassociative comultiplication $\mathcal{T} \rightarrow \mathcal{T} \otimes \mathcal{T}$. Here $\mathcal{T}$ and $\mathcal{T} \otimes \mathcal{T}$ are identified with $\mathcal{T}^{*}$ and $(\mathcal{T} \otimes \mathcal{T})^{*}$ by the bilinear form. Since $\hat{\mu}_{i}$ is associative, the dual map $\Delta_{\hat{\mu}_{i}}: \mathcal{T} \rightarrow \mathcal{T} \otimes \mathcal{T}$, $i=1,2$, of $\hat{\mu}_{i}$ becomes a coassociative multiplication. We rewrite the conditions (9), (10) and (11) in terms of the comultiplications. Then (9) and (11) are equivalent 
to the coassociativity of $\Delta_{\hat{\mu}_{i}}, i=1,2$, respectively. So we consider (10). We define a $\left(\mathcal{T}, \hat{\mu}_{1}\right)$-bimodule structure on $\mathcal{T} \otimes \mathcal{T}$ by $t \cdot(\mathcal{T} \otimes \mathcal{T}):=\left(t *_{1} \mathcal{T}\right) \otimes \mathcal{T}$ and $(\mathcal{T} \otimes \mathcal{T}) \cdot t:=\mathcal{T} \otimes\left(\mathcal{T} *_{1} t\right)$, where $t \in \mathcal{T}$ and $*_{1}$ is the associative multiplication of $\hat{\mu}_{1}$. For any $s, t, u, v \in \mathcal{T}$, we have

$$
\left(\Delta_{\hat{\mu}_{2}}\left(s *_{1} t\right) \mid u \otimes v\right)=\left(s *_{1} t \mid u *_{2} v\right),
$$

where the pairing $(-\mid-)$ is extended on $\mathcal{T} \otimes \mathcal{T}$ by the rule

$$
(s \otimes t \mid u \otimes v):=(s \mid v)(t \mid u) .
$$

The invariance property holds with respect to $\hat{\mu}_{i}, i=1,2$, for instance,

$$
\left(a *_{1} x \mid b\right)=(a * x \mid b)=(a \mid x * b)=\left(a \mid x *_{1} b\right),
$$

where $(\mathcal{A} \mid \mathcal{A})=0$ is used. Thus we have $\left(s *_{1} t \mid u *_{2} v\right)=\left(s \mid t *_{1}\left(u *_{2} v\right)\right)$. By (10), we have $t *_{1}\left(u *_{2} v\right)=\left(t *_{2} u\right) *_{1} v+\left(t *_{1} u\right) *_{2} v-t *_{2}\left(u *_{1} v\right)$. Thus (10) is equivalent to the condition

$$
\begin{aligned}
\left(\Delta_{\hat{\mu}_{2}}\left(s *_{1} t\right) \mid u \otimes v\right)= & \left(s \mid t *_{1}\left(u *_{2} v\right)\right) \\
= & \left(s \mid\left(t *_{2} u\right) *_{1} v\right)+\left(s \mid\left(t *_{1} u\right) *_{2} v\right) \\
& -\left(s \mid t *_{2}\left(u *_{1} v\right)\right) .
\end{aligned}
$$

The first term of the right-hand side of (12) is

$$
\left(s \mid\left(t *_{2} u\right) *_{1} v\right)=\left(v *_{1} s \mid t *_{2} u\right)=\left(u *_{2}\left(v *_{1} s\right) \mid t\right)=\left(u \otimes\left(v *_{1} s\right) \mid \Delta_{\hat{\mu}_{2}}(t)\right) .
$$

We put $\Delta_{\hat{\mu}_{2}}(t)=\sum t_{1} \otimes t_{2}$. Then we have

$$
\begin{aligned}
\left(u \otimes\left(v *_{1} s\right) \mid \Delta_{\hat{\mu}_{2}}(t)\right) & =\sum\left(u \mid t_{2}\right)\left(v *_{1} s \mid t_{1}\right) \\
& =\sum\left(u \mid t_{2}\right)\left(v \mid s *_{1} t_{1}\right) \\
& =\left(u \otimes v \mid s \cdot \Delta_{\hat{\mu}_{2}}(t)\right) .
\end{aligned}
$$

And the second and third terms of the right-hand side of (12) are

$$
\left(s \mid\left(t *_{1} u\right) *_{2} v\right)-\left(s \mid t *_{2}\left(u *_{1} v\right)\right)=\left(\Delta_{\hat{\mu}_{2}}(s) \mid\left(t *_{1} u\right) \otimes v\right)-\left(s *_{2} t \mid u *_{1} v\right) .
$$

We put $\Delta_{\hat{\mu}_{2}}(s)=\sum s_{1} \otimes s_{2}$. Then we have

$$
\begin{aligned}
\left(\Delta_{\hat{\mu}_{2}}(s) \mid\left(t *_{1} u\right) \otimes v\right) & =\sum\left(s_{1} \mid v\right)\left(s_{2} \mid t *_{1} u\right) \\
& =\sum\left(s_{1} \mid v\right)\left(s_{2} *_{1} t \mid u\right) \\
& =\left(\Delta_{\hat{\mu}_{2}}(s) \cdot t \mid u \otimes v\right) .
\end{aligned}
$$

and

$$
\left(s *_{2} t \mid u *_{1} v\right)=\left(\Delta_{\hat{\mu}_{1}}\left(s *_{2} t\right) \mid u \otimes v\right)=\left(\Delta_{\hat{\mu}_{1}} \circ \hat{\mu}_{2}(s, t) \mid u \otimes v\right) .
$$


From (A), (B) and (C) we obtain a compatibility condition,

$\left(\Delta_{\hat{\mu}_{2}}\left(s *_{1} t\right) \mid u \otimes v\right)=\left(s \cdot \Delta_{\hat{\mu}_{2}}(t) \mid u \otimes v\right)+\left(\Delta_{\hat{\mu}_{2}}(s) \cdot t \mid u \otimes v\right)-\left(\Delta_{\hat{\mu}_{1}} \circ \hat{\mu}_{2}(s, t) \mid u \otimes v\right)$.

Because $\mathcal{T} \otimes \mathcal{T}$ is a $\left(\mathcal{T}, \hat{\mu}_{1}\right)$-bimodule, we obtain a Hochschild complex $\left(C^{*}(\mathcal{T}, \mathcal{T} \otimes \mathcal{T}), D_{\hat{\mu}_{1}}\right)$, where $D_{\hat{\mu}_{1}}$ is a Hochschild coboundary map. Condition (13) is equivalent to (14) below. Under the assumptions of this section, the identity (10) $\left\{\hat{\mu}_{1}, \hat{\mu}_{2}\right\}=0$ is equivalent to

$$
D_{\hat{\mu}_{1}} \Delta_{\hat{\mu}_{2}}-\Delta_{\hat{\mu}_{1}} \circ \hat{\mu}_{2}=0
$$

Since $\left\{\hat{\mu}_{2}, \hat{\mu}_{1}\right\}=0$ we have $D_{\hat{\mu}_{2}} \Delta_{\hat{\mu}_{1}}-\Delta_{\hat{\mu}_{2}} \circ \hat{\mu}_{1}=0$. One can easily show that $D_{\hat{\mu}_{i}} \Delta_{\hat{\mu}_{i}}-\Delta_{\hat{\mu}_{i}} \circ \hat{\mu}_{i}=0$ holds for $i=1,2$. Thus we have $D_{\theta} \Delta_{\theta}-\Delta_{\theta} \circ \theta=0$. From (14) we have $D_{\hat{\mu}_{1}}\left(\Delta_{1} \circ \hat{\mu}_{2}\right)=0$. By direct computation, one can show that if $\mathcal{A}$ is unital (i.e. $\left.1 *_{1} \mathcal{A}=\mathcal{A} *_{1} 1\right)$, then $D_{\hat{\mu}_{1}}\left(\Delta_{1} \circ \hat{\mu}_{2}\right)=0$ implies (14).

It is obvious that $\mathcal{A}$ is a sub-coalgebra of $\left(\mathcal{T}, \Delta_{\hat{\mu}_{2}}\right)$. Since $\hat{\mu}_{2}$ is zero on $\mathcal{A} \otimes \mathcal{A}$, $\Delta_{\hat{\mu}_{2}}$ is a derivation on $\mathcal{A}$, i.e., for any $a, b \in \mathcal{A}$,

$$
\Delta_{\hat{\mu}_{2}}\left(a *_{1} b\right)=\Delta_{\hat{\mu}_{2}}(a) \cdot b+a \cdot \Delta_{\hat{\mu}_{2}}(b) \text {. }
$$

An associative and coassociative algebra $(\mathcal{I}, *, \delta)$ is called infinitesimal bialgebra ([9]) if $\delta(a * b)=a \cdot \delta(b)+\delta(b) \cdot a$ for any $a, b \in \mathcal{I}$. Thus the triple $\left(\mathcal{A}, *_{1}, \Delta_{\hat{\mu}_{2}}\right)$ is an infinitesimal bialgebra. We consider the converse. Given an infinitesimal bialgebra $(\mathcal{I}, *, \delta)$, the multiplications $*$ and $\delta$ are extended to $\mathcal{I} \oplus \mathcal{I}^{*}$ by adjoint actions. However the compatibility condition (14) is not satisfied in general. This implies that the Liezation of an infinitesimal bialgebra is not a Lie bialgebra in general. For this problem, see the detailed study by Aguiar in [3].

3.1.3. Induced dg-Lie algebras. This short section is the heart of this article. The meaning of twilled algebra is given by the proposition below. By the associative condition (9), $\left(C^{*}(\mathcal{T}), d_{\hat{\mu}_{1}}(-):=\left\{\hat{\mu}_{1},-\right\}\right)$ becomes a dg-Lie algebra. The graded space $C^{*}\left(\mathcal{A}_{2}, \mathcal{A}_{1}\right)$ is identified with an abelian subalgebra of the dg-Lie algebra via the horizontal lift. One can easily check that the derived bracket

$$
[f, g]_{\hat{\mu}_{1}}:=(-1)^{|f|-1}\left\{\left\{\hat{\mu}_{1}, f\right\}, g\right\}
$$

is closed on $C^{*}\left(\mathcal{A}_{2}, \mathcal{A}_{1}\right)$. By Lemma $2.1, C^{*}\left(\mathcal{A}_{2}, \mathcal{A}_{1}\right)$ becomes a graded Lie algebra. Further, by (10) and (11), $d_{\hat{\mu}_{2}}:=\left\{\hat{\mu}_{2},\right\}$ becomes a square zero derivation on the induced graded Lie algebra $C^{*}\left(\mathcal{A}_{2}, \mathcal{A}_{1}\right)$.

Proposition 3.3. If $\mathcal{T}=\mathcal{A}_{1} \bowtie \mathcal{A}_{2}$ is a twilled algebra, then $C^{*}\left(\mathcal{A}_{2}, \mathcal{A}_{1}\right)$ has $a$ dg-Lie algebra structure. The degree of the dg-Lie algebra structure is the same as the usual degree of cochains. 
Proof. We show only the derivation property of $d_{\hat{\mu}_{2}}$. Since $\hat{\mu}_{2}$ is an associative structure, $d_{\hat{\mu}_{2}}$ is square zero. For any cochains $f, g \in C^{*}\left(\mathcal{A}_{2}, \mathcal{A}_{1}\right)$, we have

$$
\begin{aligned}
d_{\hat{\mu}_{2}}[f, g]_{\hat{\mu}_{1}} & :=(-1)^{|f|-1}\left\{\hat{\mu}_{2},\left\{\left\{\hat{\mu}_{1}, f\right\}, g\right\}\right\} \\
& =(-1)^{|f|-1}\left\{\left\{\hat{\mu}_{2},\left\{\hat{\mu}_{1}, f\right\}\right\}, g\right\}-\left\{\left\{\hat{\mu}_{1}, f\right\},\left\{\hat{\mu}_{2}, g\right\}\right\} \\
& =(-1)^{|f|}\left\{\left\{\hat{\mu}_{1},\left\{\hat{\mu}_{2}, f\right\}\right\}, g\right\}-\left\{\left\{\hat{\mu}_{1}, f\right\},\left\{\hat{\mu}_{2}, g\right\}\right\} \\
& =\left[d_{\hat{\mu}_{2}} f, g\right]_{\hat{\mu}_{1}}+(-1)^{|f|}\left[f, d_{\hat{\mu}_{2}} g\right]_{\hat{\mu}_{1}} .
\end{aligned}
$$

By Lemma 2.1, the derived degree is given by $\operatorname{deg}_{d_{\hat{\mu}_{1}}}(f)=\operatorname{deg}(f)+1=|f|$, where $\operatorname{deg}(f)=|f|-1$ is the degree of the canonical dg-Lie algebra $\left(C^{*}(\mathcal{T}), d_{\hat{\mu}_{1}}\right)$ (recall Section 2.1). Thus $d_{\hat{\mu}_{2}}$ satisfies the defining condition (3) of a dg-Lie algebra.

Recalling deformation theory it is natural to ask: what is a solution of the MaurerCartan equation in a dg-Lie algebra? We will solve this question in Section 5.

\subsubsection{Examples}

Example 3.4 (Trivial extensions, semidirect product algebras). Let $\mathcal{A}$ be an associative algebra and let $M$ an $\mathcal{A}$-bimodule. The trivial extension $\mathcal{A} \ltimes M$ is a twilled algebra of $\mathcal{A}=\mathcal{A}_{1}$ and $M=\mathcal{A}_{2}$, where the structure $\hat{\mu}_{2}$ is trivial and $\hat{\mu}_{1}$ is defined by

$$
\hat{\mu}_{1}((a, m),(b, n)):=(a, m) *(b, n):=(a b, a \cdot n+m \cdot b),
$$

for any $(a, m),(b, n) \in \mathcal{A} \oplus M$. Here $\cdot$ is the bimodule action of $\mathcal{A}$ on $M$.

A direct product algebra $\mathcal{A} \times \mathcal{A}$ is a twilled algebra. The following example is considered as a $q$-analogue of trivial extensions.

Example 3.5 ( $q$-trivial extensions). Let $\mathcal{A}$ be an associative algebra. Define a multiplication on $\mathcal{A} \oplus \mathcal{A}$ by

$$
(a, x) *_{q}(b, y):=(a b, a y+x b+q x y),
$$

where $q \in \mathbb{K}$. Then $\left(\mathcal{A} \oplus \mathcal{A}, *_{q}\right)$ becomes a twilled algebra. We denote the twilled algebra by $\mathcal{A} \bowtie_{q} \mathcal{A}$.

If $(\mathcal{T}, \theta)$ is an associative algebra, then $C^{*}(\mathcal{T})$ becomes an associative algebra with the help of a cup product: $f \vee_{\theta} g:=\theta(f, g), f, g \in C^{*}(\mathcal{T})$.

Example 3.6. If $\mathcal{T}=\mathcal{A}_{1} \bowtie \mathcal{A}_{2}$ is a twilled algebra, then

$$
C^{*}(\mathcal{T})=C^{*}\left(\mathcal{T}, \mathcal{A}_{1} \bowtie \mathcal{A}_{2}\right) \cong C^{*}\left(\mathcal{T}, \mathcal{A}_{1}\right) \bowtie C^{*}\left(\mathcal{T}, \mathcal{A}_{2}\right)
$$

is a twilled algebra because the cup product is decomposed into $\vee_{\theta}=\vee_{\hat{\mu}_{1}}+\vee_{\hat{\mu}_{2}}$. 
3.2. Proto-, quasi-twilled algebras. A quasi-Lie bialgebra is known as a classical limit of a quasi-Hopf algebra. The notion of quasi-Lie bialgebra is generalized to proto-Lie bialgebras (see [10]). The latter is a more complicated object than quasiLie bialgebras. The proto-Lie bialgebras provide a general framework of quantumclassical correspondence. In this section, we will study associative analogues of proto-, quasi-Lie bialgebras.

Definition 3.7. Let $(\mathcal{T}, \theta)$ be an associative algebra decomposed into two subspaces, $\mathcal{T}=\mathcal{A}_{1} \oplus \mathcal{A}_{2}$. Here $\mathcal{A}_{1}$ and $\mathcal{A}_{2}$ are not necessarily subalgebras. We call the triple $\left(\mathcal{T}, \mathcal{A}_{1}, \mathcal{A}_{2}\right)$ a proto-twilled algebra.

Lemma 3.8. Let $\theta$ be an arbitrary 2-cochain in $C^{2}(\mathcal{T})$. Then $\theta$ is uniquely decomposed into four homogeneous cochains of bidegrees $0|3,1| 2,2 \mid 1$ and $3 \mid 0$,

$$
\theta=\hat{\phi}_{1}+\hat{\mu}_{1}+\hat{\mu}_{2}+\hat{\phi}_{2} \text {. }
$$

Proof. Recall the decomposition (7). The space of 2-cochains $C^{2}(\mathcal{T})$ is decomposed into

$$
C^{2}(\mathcal{T})=(0 \mid 3) \oplus(1 \mid 2) \oplus(2 \mid 1) \oplus(3 \mid 0),
$$

where $(i \mid j)$ is the space of bidegree $i \mid j$-cochains, $i, j=0,1,2,3$. The decomposition is essentially unique. Thus $\theta$ is uniquely decomposed into homogeneous cochains of bidegrees $0|3,1| 2,2 \mid 1$ and $3 \mid 0$. The four substructures $\hat{\phi}_{1}, \hat{\mu}_{1}, \hat{\mu}_{2}$ and $\hat{\phi}_{2}$ in the lemma are given as the homogeneous cochains. The proof is completed.

The multiplication $(a, x) *(b, y):=\theta((a, x),(b, y))$ of $\mathcal{T}$ is uniquely decomposed by the canonical projections $\mathcal{T} \rightarrow \mathcal{A}_{1}$ and $\mathcal{T} \rightarrow \mathcal{A}_{2}$ into the eight multiplications:

$$
\begin{aligned}
& a * b=\left(a *_{1} b, a *_{2} b\right), \\
& a * y=\left(a *_{2} y, a *_{1} y\right), \\
& x * b=\left(x *_{2} b, x *_{1} b\right), \\
& x * y=\left(x *_{1} y, x *_{2} y\right) .
\end{aligned}
$$

We put bidegrees on the four cochains: $\left\|\hat{\phi}_{1}\right\|:=0\left|3,\left\|\hat{\mu}_{1}\right\|:=1\right| 2,\left\|\hat{\mu}_{2}\right\|:=2 \mid 1$ and $\left\|\hat{\phi}_{2}\right\|:=3 \mid 0$. Then we obtain

$$
\begin{aligned}
& \hat{\phi}_{1}((a, x),(b, y))=\left(0, a *_{2} b\right), \\
& \hat{\mu}_{1}((a, x),(b, y))=\left(a *_{1} b, a *_{1} y+x *_{1} b\right), \\
& \hat{\mu}_{2}((a, x),(b, y))=\left(a *_{2} y+x *_{2} b, x *_{2} y\right), \\
& \hat{\phi}_{2}((a, x),(b, y))=\left(x *_{1} y, 0\right) .
\end{aligned}
$$

Observe that $\hat{\phi}_{1}$ and $\hat{\phi}_{2}$ are lifted cochains of $\phi_{1}(a, b):=a *_{2} b$ and $\phi_{2}(x, y):=$ $x *_{1} y$. 
Lemma 3.9. The Maurer-Cartan condition $\{\theta, \theta\}=0$ is equivalent to the following five conditions:

$$
\begin{aligned}
\left\{\hat{\mu}_{1}, \hat{\phi}_{1}\right\} & =0, \\
\frac{1}{2}\left\{\hat{\mu}_{1}, \hat{\mu}_{1}\right\}+\left\{\hat{\mu}_{2}, \hat{\phi}_{1}\right\} & =0, \\
\left\{\hat{\mu}_{1}, \hat{\mu}_{2}\right\}+\left\{\hat{\phi}_{1}, \hat{\phi}_{2}\right\} & =0, \\
\frac{1}{2}\left\{\hat{\mu}_{2}, \hat{\mu}_{2}\right\}+\left\{\hat{\mu}_{1}, \hat{\phi}_{2}\right\} & =0, \\
\left\{\hat{\mu}_{2}, \hat{\phi}_{2}\right\} & =0 .
\end{aligned}
$$

Proof. From the five conditions, one can directly check the Maurer-Cartan condition of $\theta$. We show the converse. The bidegrees of $\hat{\phi}_{1}, \hat{\mu}_{1}, \hat{\mu}_{2}$ and $\hat{\phi}_{2}$ are $0|3,1| 2,2 \mid 1$ and $3 \mid 0$, respectively. If $\{\theta, \theta\}=0$, then

$$
\begin{aligned}
\left\{\hat{\mu}_{1}, \hat{\mu}_{1}\right\}+2\left\{\hat{\mu}_{2}, \hat{\phi}_{1}\right\}+2\left\{\hat{\mu}_{1}, \hat{\mu}_{2}\right\}+2\left\{\hat{\phi}_{1}, \hat{\phi}_{2}\right\} & +\left\{\hat{\mu}_{2}, \hat{\mu}_{2}\right\}+2\left\{\hat{\mu}_{1}, \hat{\phi}_{2}\right\} \\
& +2\left\{\hat{\mu}_{1}, \hat{\phi}_{1}\right\}+2\left\{\hat{\mu}_{2}, \hat{\phi}_{2}\right\}=0 .
\end{aligned}
$$

The first two terms have 1|3-bidegree, the second two terms have 2|2-bidegree, the third two terms have $3 \mid 1$-bidegree and the last two terms have $0 \mid 4$ and $4 \mid 0$, respectively. Thus we have $\left\{\hat{\mu}_{1}, \hat{\mu}_{1}\right\}+2\left\{\hat{\mu}_{2}, \hat{\phi}_{1}\right\}=0$ for $1 \mid 3$-bidegree, which is (16). Similarly, we obtain (15)-(19).

Definition 3.10. Let $\mathcal{T}=\mathcal{A}_{1} \oplus \mathcal{A}_{2}$ be a proto-twilled algebra equipped with the structures $\left(\hat{\mu}_{1}, \hat{\mu}_{2}, \hat{\phi}_{1}, \hat{\phi}_{2}\right)$. We call the triple $\left(\mathcal{T}, \mathcal{A}_{1}, \mathcal{A}_{2}\right)$ a quasi-twilled algebra if $\phi_{2}=0$, or, equivalently, $\mathcal{A}_{2}$ is a subalgebra. Since $\mathcal{A}_{1} \oplus \mathcal{A}_{2}=\mathcal{A}_{2} \oplus \mathcal{A}_{1}$, the definition works in the case of $\phi_{2} \neq 0$ and $\phi_{1}=0$.

It is obvious that twilled algebras are special quasi-twilled algebras of $\phi_{1}=\phi_{2}=$ 0 . By Lemma 3.9, $\theta$ is the structure of a quasi-twilled algebra of $\phi_{2}=0$ if and only if

$$
\begin{aligned}
\left\{\hat{\mu}_{1}, \hat{\phi}_{1}\right\} & =0, \\
\frac{1}{2}\left\{\hat{\mu}_{1}, \hat{\mu}_{1}\right\}+\left\{\hat{\mu}_{2}, \hat{\phi}_{1}\right\} & =0, \\
\left\{\hat{\mu}_{1}, \hat{\mu}_{2}\right\} & =0, \\
\frac{1}{2}\left\{\hat{\mu}_{2}, \hat{\mu}_{2}\right\} & =0 .
\end{aligned}
$$

In Proposition 3.3, we saw that $C^{*}\left(\mathcal{A}_{2}, \mathcal{A}_{1}\right)$ has a dg-Lie algebra structure. In the quasi-twilled algebra case, by (23), $d_{\hat{\mu}_{2}}$ is still a square zero derivation, but the derived bracket by $\hat{\mu}_{1}$ does not satisfy the graded Jacobi identity in general. However the Jacobiator still satisfies a weak Jacobi identity in the sense of homotopy Lie algebras ([6], [15]). The 3-cochain $\frac{1}{2}\left\{\hat{\mu}_{1}, \hat{\mu}_{1}\right\}$ rises up to the graded Jacobiator via the derived 
bracket,

$$
\begin{aligned}
& (-1)^{|g|-1} \frac{1}{2}\left\{\left\{\left\{\left\{\hat{\mu}_{1}, \hat{\mu}_{1}\right\}, f\right\}, g\right\}, h\right\} \\
& \quad=\left[f,[g, h]_{\hat{\mu}_{1}}\right]_{\hat{\mu}_{1}}-\left[[f, g]_{\hat{\mu}_{1}}, h\right]_{\hat{\mu}_{1}}-(-1)^{|f \| g|}\left[g,[f, h]_{\hat{\mu}_{1}}\right]_{\hat{\mu}_{1}} .
\end{aligned}
$$

By (21), the Jacobiator is also given by $-\left\{\hat{\mu}_{2}, \hat{\phi}_{1}\right\}$. We define a tri-linear bracket product (homotopy) on $C^{*}\left(\mathcal{A}_{2}, \mathcal{A}_{1}\right)$ by

$$
[f, g, h]_{\hat{\phi}_{1}}:=(-1)^{|g|-1}\left\{\left\{\left\{\hat{\phi}_{1}, f\right\}, g\right\}, h\right\} .
$$

Since $C^{*}\left(\mathcal{A}_{2}, \mathcal{A}_{1}\right)$ is abelian with respect to $\{-,-\}$, the tribracket is skew-symmetric. We can show that the system $\left(d_{\hat{\mu}_{2}},[\cdot, \cdot]_{\hat{\mu}_{1}},[\cdot, \cdot, \cdot]_{\hat{\phi}_{1}}\right)$ defines a strong homotopy Lie algebra structure of $l_{n \geq 4}:=0$ on $C^{*}\left(\mathcal{A}_{2}, \mathcal{A}_{1}\right)$. This assertion is shown in [24] as a corollary of a more general result.

The complex plane, $\mathcal{T}:=\mathbb{C}$, is a quasi-twilled algebra decomposed into the real part and the imaginary part. Given an $\mathbb{R}$-algebra $\mathcal{A}$, the complexification $\mathbb{C} \otimes_{\mathbb{R}} \mathcal{A}=$ $\mathcal{A} \oplus \sqrt{-1} \mathcal{A}$ is a quasi-twilled algebra.

Example 3.11 (Quasi-trivial extension). Let $\mathcal{A}$ be an associative algebra. Define a multiplication on $\mathcal{A} \oplus \mathcal{A}$ by

$$
(a, x) *_{Q}(b, y):=(a b+Q x y, a y+x b),
$$

where $Q \in \mathbb{K}$. Then $\mathcal{A} \oplus \mathcal{A}$ becomes a quasi-twilled algebra, where $\phi_{2}(x, y):=$ $Q x y$. We denote this algebra by $\mathcal{A} \oplus Q \mathcal{A}$.

\section{Twisting by a 1-cochain}

Let $h$ be a 1-cochain in $C^{1}(\mathcal{T})$. By analogy with Hamiltonian vector fields, we define an operator by $X_{h}:=\{\cdot, h\}$, and by analogy with Hamiltonian flows, we put

$$
\exp \left(X_{h}\right)(\cdot):=1+X_{h}+\frac{1}{2 !} X_{h}^{2}+\frac{1}{3 !} X_{h}^{3}+\ldots,
$$

where $X_{h}^{2}:=\{\{\cdot, h\}, h\}$ and $X_{h}^{n}$ is defined in the same manner. Observe that $\exp \left(X_{h}\right)$ is not well defined in general.

Let $\left(\mathcal{T}=\mathcal{A}_{1} \oplus \mathcal{A}_{2}, \theta\right)$ be a proto-twilled algebra, and let $\hat{H} \in C^{1}(\mathcal{T})$ be the lift of a linear map $H: \mathcal{A}_{2} \rightarrow \mathcal{A}_{1}\left(\right.$ or $\left.H: \mathcal{A}_{1} \rightarrow \mathcal{A}_{2}\right)$. Then $\exp \left(X_{\hat{H}}\right)$ is always well defined as an operator because $\hat{H} \hat{H}=0$ (recall Lemma 2.2).

Definition 4.1. A transformation

$$
\theta^{H}:=\exp \left(X_{\widehat{H}}\right)(\theta)
$$

is called a twisting of $\theta$ by $H$. 
It is clear that the result of twisting by $H$ is again a 2-cochain. We can consider the twisting operations as special examples of gauge transformations in deformation theory (see [6]). Lemma 4.2 and Proposition 4.3 follow from standard arguments in deformation theory.

Lemma 4.2. $\theta^{H}=e^{-\widehat{H}} \theta\left(e^{\widehat{H}} \otimes e^{\widehat{H}}\right)$, where $e^{ \pm \hat{H}}=1 \pm \hat{H}$.

Proof. We have

$$
\begin{aligned}
e^{-\hat{H}} \theta\left(e^{\widehat{H}} \otimes e^{\hat{H}}\right)= & \theta\left(e^{\hat{H}} \otimes e^{\hat{H}}\right)-\hat{H} \theta\left(e^{\hat{H}} \otimes e^{\hat{H}}\right) \\
= & +\theta(1 \otimes \hat{H})+\theta(\hat{H} \otimes 1)+\theta(\hat{H} \otimes \hat{H})-\hat{H} \theta \\
& \quad-\hat{H} \theta(1 \otimes \hat{H})-\hat{H} \theta(\hat{H} \otimes 1)-\hat{H} \theta(\hat{H} \otimes \hat{H}) \\
= & +\theta(1 \otimes \hat{H})+\theta(\hat{H} \otimes 1)-\hat{H} \theta+\theta(\hat{H} \otimes \hat{H}) \\
& \quad-\hat{H} \theta(1 \otimes \hat{H})-\hat{H} \theta(\hat{H} \otimes 1)-\hat{H} \theta(\hat{H} \otimes \hat{H}) .
\end{aligned}
$$

Since $\hat{H} \hat{H}=0$, for any $I \geq 4$, we have $X_{\widehat{H}}^{I}(\theta)=0$. Thus we have

$$
\exp \left(X_{\widehat{H}}\right)(\theta)=\theta+\{\theta, \hat{H}\}+\frac{1}{2}\{\{\theta, \hat{H}\}, \hat{H}\}+\frac{1}{6}\{\{\{\theta, \hat{H}\}, \hat{H}\}, \hat{H}\} .
$$

One can directly check the three identities below.

$$
\begin{aligned}
\{\theta, \hat{H}\} & =\theta(\hat{H} \otimes 1)+\theta(1 \otimes \hat{H})-\hat{H} \theta, \\
\frac{1}{2}\{\{\theta, \hat{H}\}, \hat{H}\} & =\theta(\hat{H} \otimes \hat{H})-\hat{H} \theta(\hat{H} \otimes 1)-\hat{H} \theta(1 \otimes \hat{H}), \\
\frac{1}{6}\{\{\{\theta, \hat{H}\}, \hat{H}\}, \hat{H}\} & =-\hat{H} \theta(\hat{H} \otimes \hat{H}) .
\end{aligned}
$$

The proof of the lemma is completed.

From the above lemma, we have $\left\{\theta^{H}, \theta^{H}\right\}=e^{-H}\{\theta, \theta\}\left(e^{\widehat{H}} \otimes e^{\widehat{H}} \otimes e^{\widehat{H}}\right)$. This implies

Proposition 4.3. The result of twisting $\theta^{H}$ is an associative structure, i.e., one has $\left\{\theta^{H}, \theta^{H}\right\}=0$.

The following corollary is useful.

Corollary 4.4. The twisting by $H$ induces an algebra isomorphism

$$
e^{H}:\left(\mathcal{T}, \theta^{H}\right) \rightarrow(\mathcal{T}, \theta) .
$$

Obviously, $\left(\mathcal{T}, \theta^{H}\right)$ is also a proto-twilled algebra. Thus $\theta^{H}$ is also decomposed into the unique four substructures. The twisting operations are completely determined by the following result. 
Theorem 4.5. Assume a decomposition of $\theta, \theta:=\hat{\mu}_{1}+\hat{\mu}_{2}+\hat{\phi}_{1}+\hat{\phi}_{2}$. The unique four substructures of $\theta^{H}$ have the form

$$
\begin{aligned}
\hat{\phi}_{1}^{H} & =\hat{\phi}_{1}, \\
\hat{\mu}_{1}^{H} & =\hat{\mu}_{1}+\left\{\hat{\phi}_{1}, \hat{H}\right\}, \\
\hat{\mu}_{2}^{H} & =\hat{\mu}_{2}+d_{\hat{\mu}_{1}} \hat{H}+\frac{1}{2}\left\{\left\{\hat{\phi}_{1}, \hat{H}\right\}, \hat{H}\right\}, \\
\hat{\phi}_{2}^{H} & =\hat{\phi}_{2}+d_{\hat{\mu}_{2}} \hat{H}+\frac{1}{2}[\hat{H}, \hat{H}]_{\hat{\mu}_{1}}+\frac{1}{6}\left\{\left\{\left\{\hat{\phi}_{1}, \hat{H}\right\}, \hat{H}\right\}, \hat{H}\right\},
\end{aligned}
$$

where $d_{\hat{\mu}_{i}}(-):=\left\{\hat{\mu}_{i},-\right\},(i=1,2)$ and $[\hat{H}, \hat{H}]_{\hat{\mu}_{1}}:=\left\{\left\{\hat{\mu}_{1}, \hat{H}\right\}, \hat{H}\right\}$.

Proof. The first term of $\exp \left(X_{\hat{H}}\right)(\theta)$ is $\theta$. It follows from bidegree calculus that $\left\{\hat{\phi}_{2}, \hat{H}\right\}=0$ because $\left\|\hat{\phi}_{2}\right\|=3 \mid 0$ and $\|\hat{H}\|=2 \mid 0$. Thus the second term of $\exp \left(X_{\hat{H}}\right)(\theta)$ has the form

$$
\left\{\hat{\mu}_{1}, \hat{H}\right\}+\left\{\hat{\mu}_{2}, \hat{H}\right\}+\left\{\hat{\phi}_{1}, \hat{H}\right\} .
$$

We have $\left\|\left\{\hat{\mu}_{1}, \hat{H}\right\}\right\|=2\left|1,\left\|\left\{\hat{\mu}_{2}, \hat{H}\right\}\right\|=3\right| 0$ and $\left\|\left\{\hat{\phi}_{1}, \hat{H}\right\}\right\|=1 \mid 2$, which implies $\left\{\left\{\hat{\mu}_{2}, \widehat{H}\right\}, \hat{H}\right\}=0$. Thus the third term has the form

$$
\frac{1}{2}\left(\left\{\left\{\hat{\mu}_{1}, \hat{H}\right\}, \hat{H}\right\}+\left\{\left\{\hat{\phi}_{1}, \hat{H}\right\}, \hat{H}\right\}\right) .
$$

The bidegrees are $\left\|\left\{\left\{\hat{\mu}_{1}, \hat{H}\right\}, \hat{H}\right\}\right\|=3 \mid 0$ and $\left\|\left\{\left\{\hat{\phi}_{1}, \hat{H}\right\}, \hat{H}\right\}\right\|=2 \mid 1$. The final term is $\{\{\{\theta, \hat{H}\}, \widehat{H}\}, \widehat{H}\}=\left\{\left\{\left\{\hat{\phi}_{1}, \hat{H}\right\}, \hat{H}\right\}, \widehat{H}\right\}$, which has the bidegree $3 \mid 0$. Thus the sum of all $3 \mid 0$-terms is

$$
\hat{\phi}_{2}+\left\{\hat{\mu}_{2}, \hat{H}\right\}+\frac{1}{2 !}\left\{\left\{\hat{\mu}_{1}, \hat{H}\right\}, \hat{H}\right\}+\frac{1}{3 !}\left\{\left\{\left\{\hat{\phi}_{1}, \hat{H}\right\}, \hat{H}\right\}, \hat{H}\right\},
$$

which gives (28). In this way, the remaining three conditions hold.

\section{Maurer-Cartan equations}

Let $\mathcal{T}=\mathcal{A}_{1} \oplus \mathcal{A}_{2}$ be a proto-twilled algebra equipped with an associative structure $\theta$ and let $\left(\hat{\phi}_{1}, \hat{\mu}_{1}, \hat{\mu}_{2}, \hat{\phi}_{2}\right)$ be the unique four substructures of $\theta$. In this section we discuss various examples of twisting operations.

5.1. The case of $\phi_{1}=\mathbf{0}$ and $\phi_{2}=\mathbf{0}$. Then $\mathcal{T}=\mathcal{A}_{1} \bowtie \mathcal{A}_{2}$ is a twilled algebra. However the result of twisting by $H: \mathcal{A}_{2} \rightarrow \mathcal{A}_{1},\left(\mathcal{T}_{H}, \mathcal{A}_{1}, \mathcal{A}_{2}\right)$, is a quasi-twilled algebra in general. The twisted structures have the form

$$
\begin{aligned}
\hat{\mu}_{1}^{H} & =\hat{\mu}_{1}, \\
\hat{\mu}_{2}^{H} & =\hat{\mu}_{2}+d_{\hat{\mu}_{1}} \hat{H}, \\
\hat{\phi}_{2}^{H} & =d_{\hat{\mu}_{2}} \hat{H}+\frac{1}{2}[\hat{H}, \hat{H}]_{\hat{\mu}_{1}} .
\end{aligned}
$$


This $\hat{\phi}_{2}^{H}$ is called curvature. The derivation operator $d_{\hat{\mu}_{2}}$ on the graded Lie algebra $C^{*}\left(\mathcal{A}_{2}, \mathcal{A}_{1}\right)$ is modified by $\left.H, d_{\hat{\mu}_{2}^{H}}(-)=d_{\hat{\mu}_{2}}(-)+[\hat{H},-]\right]_{\hat{\mu}_{1}}$, where $d_{\hat{\mu}_{2}^{H}} d_{\hat{\mu}_{2}^{H}} \neq 0$ in general. By Lemma $3.9(19)$, the cocycle condition of $\phi_{2}^{H}$ still holds:

$$
d_{\hat{\mu}_{2}^{H}} \hat{\phi}_{2}^{H}=0 .
$$

This is a kind of Bianchi identity.

5.1.1. Maurer-Cartan operators. In Proposition 3.3 we saw that $C^{*}\left(\mathcal{A}_{2}, \mathcal{A}_{1}\right)$ has a dg-Lie algebra structure. We study the Maurer-Cartan equation in this dg-Lie algebra.

Corollary 5.1. The result of twisting $\mathcal{T}_{H}=\mathcal{A}_{1} \oplus \mathcal{A}_{2}$ is also a twilled algebra if and only if the curvature vanishes, or, equivalently, $H$ is a solution of the Maurer-Cartan equation

$$
d_{\hat{\mu}_{2}} \hat{H}+\frac{1}{2}[\hat{H}, \hat{H}]_{\hat{\mu}_{1}}=0 .
$$

The condition (MC) is equivalent to

$$
H(x) *_{1} H(y)+H(x) *_{2} y+x *_{2} H(y)=H\left(H(x) *_{1} y+x *_{1} H(y)\right)+H\left(x *_{2} y\right) .
$$

Proof. We have $d_{\hat{\mu}_{2}} \hat{H}=\hat{\mu}_{2}(\hat{H} \otimes 1)-\hat{H} \hat{\mu}_{2}+\hat{\mu}_{2}(1 \otimes \hat{H})$ and

$$
\frac{1}{2}[\hat{H}, \hat{H}]_{\hat{\mu}_{1}}=\frac{1}{2}\left\{\left\{\hat{\mu}_{1}, \hat{H}\right\}, \hat{H}\right\}=\hat{\mu}_{1}(\hat{H} \otimes \hat{H})-\hat{H} \hat{\mu}_{1}(1 \otimes \hat{H})-\hat{H} \hat{\mu}_{1}(\hat{H} \otimes 1) .
$$

This gives

$$
\begin{aligned}
\left(d_{\hat{\mu}_{2}} \hat{H}+\frac{1}{2}[\hat{H}, \hat{H}]_{\hat{\mu}_{1}}\right)((a, x),(b, y))= & H(x) *_{2} y-H\left(x *_{2} y\right)+x *_{2} H(y) \\
& +H(x) *_{1} H(y)-H\left(H(x) *_{1} y\right. \\
& \left.+x *_{1} H(y)\right)
\end{aligned}
$$

for any $(a, x),(b, y) \in \mathcal{T}$.

Definition 5.2. Let $\mathcal{A}_{1} \bowtie \mathcal{A}_{2}$ be a twilled algebra and let $H: \mathcal{A}_{2} \rightarrow \mathcal{A}_{1}$ a linear map. We call the operator $H$ in (MC), or, equivalently, in (29) Maurer-Cartan operator. The Maurer-Cartan operator is called strong if it is a derivation with respect to the multiplication $*_{2}$, i.e.,

$$
H\left(x *_{2} y\right)=x *_{2} H(y)+H(x) *_{2} y .
$$

In [16] the Maurer-Cartan equation in other dg-Lie algebras was studied. The concept of strong solution is due to their work. If $H$ is strong, then the identity, 
$H(x) *_{1} H(y)=H\left(H(x) *_{1} y+x *_{1} H(y)\right)$, automatically holds. The strong Maurer-Cartan condition is equivalent to

$$
d_{\hat{\mu}_{2}} \hat{H}=\frac{1}{2}[\hat{H}, \hat{H}]_{\hat{\mu}_{1}}=0 .
$$

We easily obtain

Corollary 5.3. If $H$ is the Maurer-Cartan operator, then

$$
x \times_{H} y:=H(x) *_{1} y+x *_{1} H(y)+x *_{2} y
$$

is an associative multiplication on $\mathcal{A}_{2}$.

Proof. When $H$ satisfies (MC), we have $\hat{\phi}_{2}^{H}=0$. By Lemma 3.9, we obtain $\left\{\hat{\mu}_{2}^{H}, \hat{\mu}_{2}^{H}\right\}=0$, which gives the associativity of $\hat{\mu}_{2}^{H}$. The multiplication has the following form on $\mathcal{A}_{2}$ :

$$
\hat{\mu}_{2}^{H}(x, y)=H(x) *_{1} y+x *_{1} H(y)+x *_{2} y .
$$

We recall Rota-Baxter operators mentioned in the Introduction.

Example 5.4 (Rota-Baxter operators of weight $q$ ). Let $\mathcal{A}$ be an associative algebra. Recall the twilled algebra of Example 3.5. The multiplication of $\mathcal{A}_{\Delta} \triangle_{q}$ is defined by

$$
(a, x) *_{q}(b, y):=(a b, a y+x b+q x y),
$$

where $q \in \mathbb{K}$ (weight). By (29), the Maurer-Cartan operators on $\mathcal{A} \bowtie_{q} \mathcal{A}$ satisfy the Rota-Baxter identity of weight $q$,

$$
R(x) R(y)=R(R(x) y+x R(y))+q R(x y),
$$

where we put $R:=H$. Thus Rota-Baxter operators can be seen as examples of Maurer-Cartan operators.

As an example of Rota-Baxter operator, we know

$$
R(f)(x):=f(q x)+f\left(q^{2} x\right)+f\left(q^{3} x\right)+\cdots
$$

where $R$ is defined on a certain algebra of functions (see [19]).

5.1.2. The case of $\hat{\mu}_{\mathbf{2}}=\mathbf{0}$. In this case, since $d_{\hat{\mu}_{2}}=0$, the Maurer-Cartan equation simply has the form $[\hat{H}, \hat{H}]_{\hat{\mu}_{1}} / 2=0$, or, equivalently, (29) reduces to the identity

$$
H(x) *_{1} H(y)=H\left(H(x) *_{1} y+x *_{1} H(y)\right) .
$$

Furthermore, if $\mathcal{A}_{2}=\mathcal{A}_{1}$ as a canonical bimodule, then $H$ is considered a RotaBaxter operator with weight zero. 
Definition 5.5 ([23]). Let $\mathcal{A}$ be an associative algebra and let $M$ be an $\mathcal{A}$-bimodule. A linear map $\pi: M \rightarrow \mathcal{A}$ is called generalized Rota-Baxter operator (of weight zero) if $\pi$ is a solution of the identity

$$
\pi(m) \pi(n)=\pi(\pi(m) \cdot n+m \cdot \pi(n)),
$$

or, equivalently, $[\hat{\pi}, \hat{\pi}] \hat{\mu} / 2=0$, where $m, n \in M$ and $\hat{\mu}$ is the associative structure of $\mathcal{A} \ltimes M$.

A generalized Rota-Baxter operator is obviously a (strong) Maurer-Cartan operator. Given a generalized Rota-Baxter operator $\pi: M \rightarrow \mathcal{A}$, we have a twilled algebra $\mathcal{A} \bowtie M_{\pi}$ by the twisting of $\mathcal{A} \ltimes M$ by $\pi$, where $M_{\pi}$ is an associative subalgebra given by Corollary 5.3. The associative structure of $\mathcal{A} \bowtie M_{\pi}$ is the sum $\hat{\mu}+\{\hat{\mu}, \hat{\pi}\}$ of two structures.

Corollary 5.6. Under the assumptions above, if $\pi_{1}$ is a second generalized RotaBaxter operator on $\mathcal{A} \ltimes M$, i.e., $\left[\hat{\pi}_{1}, \hat{\pi}_{1}\right]_{\hat{\mu}}=0$, then $H:=\pi_{1}-\pi$ is the MaurerCartan operator on $\mathcal{A} \bowtie M_{\pi}$. If $H$ is strong, then $\pi+t H$ is a one-parameter family of generalized Rota-Baxter operators for any $t \in \mathbb{K}$.

Proof. By assumption, we have $[\hat{H}, \hat{H}]_{\hat{\mu}} / 2=-\left[\hat{\pi}_{1}, \hat{\pi}\right]_{\hat{\mu}}$. On the other hand, since $d_{\hat{\mu}_{2}}(\cdot)=\{\{\hat{\mu}, \hat{\pi}\}, \cdot\}$, we have

$$
d_{\hat{\mu}_{2}} \hat{H}=\left\{\{\hat{\mu}, \hat{\pi}\}, \hat{\pi}_{1}\right\}=\left[\hat{\pi}, \hat{\pi}_{1}\right]_{\hat{\mu}}=\left[\hat{\pi}_{1}, \hat{\pi}\right]_{\hat{\mu}} .
$$

We obtain the condition (MC). Thus Maurer-Cartan operators on $\mathcal{A} \bowtie M_{\pi}$ are given as the difference of $\pi$ with generalized Rota-Baxter operators. If $H$ is a strong Maurer-Cartan operator, then so is $t H$ for any $t \in \mathbb{K}$. This implies the second part of the corollary.

We recall Section 3.1.2. Let $\mathcal{A}$ be a finite dimensional associative algebra and let $\mathcal{A}^{*}$ be the dual space. By canonical adjoint action, $\mathcal{A}$ acts on the dual space. In this case, there are interesting similarities between generalized Rota-Baxter operators and classical $r$-matrices. We recall the classical Yang-Baxter equation (CYBE). There exist several equivalent definitions of CYBE. Here is one of them: CYBE is defined to be an operator identity in the category of Lie algebras,

$$
[\tilde{r}(x), \tilde{r}(y)]=\tilde{r}([\tilde{r}(x), y]+[x, \tilde{r}(y)]),
$$

where $r$ is a 2-tensor in $\mathfrak{g} \otimes \mathfrak{g}$ ( $\mathfrak{g}$ is a finite dimensional Lie algebra), $\tilde{r}: \mathfrak{g}^{*} \rightarrow \mathfrak{g}$ is the associated linear map, $x, y$ are elements in the dual space $\mathrm{g}^{*}$ and the brackets on the right-hand side are adjoint actions. The space of alternative tensors $\wedge^{*} \mathrm{~g}$ has a graded Lie algebra structure of the Schouten bracket. If $r$ is an element in $\mathrm{g} \wedge \mathrm{g}$, then the Schouten bracket $[r, r]$ is in $\bigwedge^{3} \mathrm{~g}$, and $[r, r]=0$ if and only if $\tilde{r}$ satisfies CYBE above. Such a matrix $r$ is called triangular $r$-matrix. When $g$ is a Lie algebroid, a 
triangular $r$-matrix is a Poisson structure. The notion of generalized Rota-Baxter operator can be seen as an associative version of triangular $r$-matrices and Poisson structures. We believe that this picture is justified by the following example.

Example 5.7. Let $\mathcal{A}$ be a 2-dimensional algebra generated by $\left(\begin{array}{ll}0 & 1 \\ 0 & 0\end{array}\right)$ and $\left(\begin{array}{ll}1 & 0 \\ 0 & 0\end{array}\right)$. The dual space $\mathcal{A}^{*}$ is an $\mathcal{A}$-bimodule by adjoint action. Thus we have a twilled algebra $\mathcal{A} \ltimes \mathcal{A}^{*}$. Define a tensor $r$ by

$$
r:=\left(\begin{array}{ll}
0 & 1 \\
0 & 0
\end{array}\right) \wedge\left(\begin{array}{ll}
1 & 0 \\
0 & 0
\end{array}\right) .
$$

This tensor is identified to a map $\tilde{r}: \mathcal{A}^{*} \rightarrow \mathcal{A}$. By direct computation, one can check that the map is a generalized Rota-Baxter operator.

In general, if a 2-tensor $r \in \mathcal{A} \wedge \mathcal{A}$ satisfies Aguiar's multiplicative equation (called associative Yang-Baxter equation in [1], [2], [3])

$$
r_{13} r_{12}-r_{12} r_{23}+r_{23} r_{13}=0,
$$

then $\tilde{r}: \mathcal{A}^{*} \rightarrow \mathcal{A}$ is a generalized Rota-Baxter operator (see [23]). Conversely, a skew-symmetric generalized Rota-Baxter operator satisfies (AYBE) above. In non skew-symmetric cases, there is a delicate difference between AYBE and the generalized Rota-Baxter condition.

When $r$ is skew-symmetric, the twisting by $r$ preserves the bilinear pairing $(-\mid-)$ in Section 3.1.2. Thus the associative structure $\hat{\mu}+\{\hat{\mu}, \hat{r}\}$ satisfies the invariant condition in the sense of 3.1.2.

A Poisson structure is considered as a sheaf version of triangular matrices. It is natural to ask what is a sheaf version of Rota-Baxter operators. We do not yet have an interesting solution. We wish to find a Rota-Baxter operator on the universal enveloping algebra of a Lie algebroid. If there exists such a Rota-Baxter operator, it is considered as an example of the sheaf version.

5.2. The case of $\phi_{1} \neq \mathbf{0}$ and $\phi_{2}=\mathbf{0}$. Then $\mathcal{T}=\mathcal{A}_{1} \oplus \mathcal{A}_{2}$ is a quasi-twilled algebra. However $\mathcal{T}_{H}=\mathscr{A}_{1} \oplus \mathcal{A}_{2}$ is not necessarily a quasi-twilled algebra because $\phi_{1}^{H}=\phi_{1} \neq 0$ and

$$
\hat{\phi}_{2}^{H}=d_{\hat{\mu}_{2}} \hat{H}+\frac{1}{2}[\hat{H}, \hat{H}]_{\hat{\mu}_{1}}+\frac{1}{6}\left\{\left\{\left\{\hat{\phi}_{1}, \hat{H}\right\}, \hat{H}\right\}, \hat{H}\right\} \neq 0 .
$$

In general, the results of twisting have the form

$$
\begin{aligned}
\hat{\phi}_{1}^{H} & =\hat{\phi}_{1}, \\
\hat{\mu}_{1}^{H} & =\hat{\mu}_{1}+\left\{\hat{\phi}_{1}, \hat{H}\right\}, \\
\hat{\mu}_{2}^{H} & =\hat{\mu}_{2}+d_{\hat{\mu}_{1}} \hat{H}+\frac{1}{2}\left\{\left\{\hat{\phi}_{1}, \hat{H}\right\}, \hat{H}\right\}, \\
\hat{\phi}_{2}^{H} & =d_{\hat{\mu}_{2}} \hat{H}+\frac{1}{2}[\hat{H}, \hat{H}]_{\hat{\mu}_{1}}+\frac{1}{6}\left\{\left\{\left\{\hat{\phi}_{1}, \hat{H}\right\}, \hat{H}\right\}, \hat{H}\right\} .
\end{aligned}
$$


Since $\hat{\mu}_{1}$ is not associative, the derived bracket $[\cdot, \cdot]_{\hat{\mu}_{1}}$ does not satisfy the graded Jacobi rule in general. However the space $C^{*}\left(\mathcal{A}_{2}, \mathcal{A}_{1}\right)$ still has a homotopy Lie algebra structure $\left(d_{\hat{\mu}_{2}},[\cdot, \cdot]_{\hat{\mu}_{1}},[\cdot, \cdot, \cdot]_{\hat{\phi}_{1}}\right)$ of Section 3.2. We consider the MaurerCartan equation in this homotopy Lie algebra. The following two corollaries are proved in the same way as Corollary 5.1 and Corollary 5.3.

Corollary 5.8. The result of twisting $\mathcal{T}_{H}=\mathcal{A}_{1} \oplus \mathcal{A}_{2}$ is also a quasi-twilled algebra if and only if it is a solution of the twisted Maurer-Cartan equation

$$
d_{\hat{\mu}_{2}} \hat{H}+\frac{1}{2}[\hat{H}, \hat{H}]_{\hat{\mu}_{1}}+\frac{1}{6}[\hat{H}, \hat{H}, \hat{H}]_{\hat{\phi}_{1}}=0,
$$

or, equivalently,

$$
\begin{aligned}
& H(x) *_{1} H(y)+H(x) *_{2} y+x *_{2} H(y) \\
& \quad=H\left(H(x) *_{1} y+x *_{1} H(y)\right)+H\left(x *_{2} y\right)+H\left(\phi_{1}(H(x), H(y))\right)
\end{aligned}
$$

for any $x, y \in \mathcal{A}_{2}$.

Corollary 5.9. If $\mathcal{T}_{H}=\mathcal{A}_{1} \oplus \mathcal{A}_{2}$ is a quasi-twilled algebra, then $x \times_{H, \phi_{1}} y:=\hat{\mu}_{2}^{H}(x, y)=H(x) *_{1} y+x *_{1} H(y)+x *_{2} y+\phi_{1}(H(x), H(y))$. is an associative multiplication on $\mathcal{A}_{2}$.

Example 5.10 (Twisted Rota-Baxter operators [23]). If $\hat{\mu}_{2}=0$, or $*_{2}$ is trivial, then (32) is reduced to an identity:

$$
H(x) *_{1} H(y)=H\left(H(x) *_{1} y+x *_{1} H(y)\right)+H\left(\phi_{1}(H(x), H(y))\right) .
$$

(TRB1) is equivalent to

$$
\frac{1}{2}[\hat{H}, \hat{H}]_{\hat{\mu}_{1}}=-\frac{1}{6}[\hat{H}, \hat{H}, \hat{H}]_{\hat{\phi}_{1}} .
$$

Such an operator $H$ is called twisted Rota-Baxter operator (of weight zero).

As an example of twisted Rota-Baxter operators, we mention the Reynolds operators in probability theory ([20]). Let $\mathcal{A}$ be a certain functional algebra. Define an operator $R: \mathcal{A} \rightarrow \mathcal{A}$ by

$$
R(f)(x):=\int_{0}^{\infty} e^{-t} f(x-t) d t .
$$

Then $R$ satisfies the identity

$$
R(f) R(g)=R(R(f) g+f R(g))-R(R(f) R(g)) .
$$

Such an operator is called Reynolds operator. The last term $-R(R(f) R(g))=$ $R \phi(R(f), R(g))$ can be seen as the cocycle term of twisted Rota-Baxter identity. Thus a Reynolds operator can be seen as a homotopy version of Rota-Baxter operators of weight zero. 
A Reynolds operator is used, in the study of turbulent flow, in order to induce a mean field model of Navier-Stokes equation (so-called Reynolds equation). One can easily verify that if $R(f):=\bar{f}$ is the mean of $f$, then the operator satisfies the identity above, because an averaging operation satisfies the identities $\overline{\bar{f} g}=\bar{f} \cdot \bar{g}=\overline{f \bar{g}}$ and $\overline{\bar{f}}=\bar{f}$ in general. Unfortunately, we do not know of an application of our construction to Rota's theory.

5.3. The case of $\phi_{1}=\mathbf{0}$ and $\phi_{2} \neq \mathbf{0}$. In this case, $\hat{\phi}_{1}=\hat{\phi}_{1}^{H}=0$, and thus $\hat{\mu}_{1}$ and $\hat{\mu}_{1}^{H}$ are both associative. The twisted four substructures have the form

$$
\begin{aligned}
& \hat{\mu}_{1}^{H}=\hat{\mu}_{1}, \\
& \hat{\mu}_{2}^{H}=\hat{\mu}_{2}+d_{\hat{\mu}_{1}} \hat{H}, \\
& \hat{\phi}_{2}^{H}=\hat{\phi}_{2}+d_{\hat{\mu}_{2}} \hat{H}+\frac{1}{2}[\hat{H}, \hat{H}]_{\hat{\mu}_{1}} .
\end{aligned}
$$

Similar to Corollary 5.1 and Corollary 5.3, we obtain the two corollaries below.

Corollary 5.11. The result of twisting $\mathcal{T}_{H}=\mathcal{A}_{1} \oplus \mathcal{A}_{2}$ is a usual twilled algebra, i.e., $\hat{\phi}_{2}^{H}=0$ if and only if $H$ is a solution of the quasi-Maurer-Cartan equation

$$
d_{\hat{\mu}_{2}} \hat{H}+\frac{1}{2}[\hat{H}, \hat{H}]_{\hat{\mu}_{1}}=-\hat{\phi}_{2},
$$

or, equivalently,

$$
\begin{aligned}
H(x) *_{2} y+ & x *_{2} H(y)+H(x) *_{1} H(y)+\phi_{2}(x, y) \\
& =H\left(H(x) *_{1} y+x *_{1} H(y)\right)+H\left(x *_{2} y\right) .
\end{aligned}
$$

Corollary 5.12. If $H$ satisfies (QMC), then $\hat{\mu}_{2}^{H}$ is an associative structure and defines an associative multiplication on $\mathcal{A}_{2}$ by

$$
x \times_{H, \phi_{2}} y:=\hat{\mu}_{2}^{H}(x, y)=H(x) *_{1} y+x *_{1} H(y)+x *_{2} y .
$$

We consider the case of $\hat{\mu}_{2}=0$. Then (QMC) and (33) reduce to the identities

$$
\frac{1}{2}[\hat{H}, \hat{H}]_{\hat{\mu}_{1}}=-\hat{\phi}_{2}
$$

and

$$
H(x) *_{1} H(y)-H\left(H(x) *_{1} y+x *_{1} H(y)\right)=-\phi_{2}(x, y),
$$

respectively.

Recall the quasi-twilled algebra $\mathcal{A} \oplus Q \mathcal{A}$ of Example 3.11.

Claim. Define a linear map $(a, x) \mapsto\left(\frac{q}{2} x, 0\right)$ on $\mathcal{A} \oplus \mathcal{A}$. Then its integral $e^{\widehat{q / 2}}$ is an algebra isomorphism

$$
e^{\widehat{q / 2}}: \mathcal{A} \bowtie_{q} \mathcal{A} \rightarrow \mathcal{A} \oplus Q \mathcal{A}, \quad Q=\frac{q^{2}}{4} .
$$


Proof. We have

$$
\begin{aligned}
e^{\widehat{q / 2}}\left((a, x) *_{q}(b, y)\right) & =\left(a b+\frac{q}{2} a y+\frac{q}{2} x b+\frac{q^{2}}{2} x y, a y+x b+q x y\right) \\
& =\left(\left(a+\frac{q}{2} x\right)\left(b+\frac{q}{2} y\right)+\frac{q^{2}}{4} x y, a y+x b+q x y\right) \\
& =\left(a+\frac{q}{2} x, x\right) *_{Q}\left(b+\frac{q}{2} y, y\right), \quad Q=\frac{q^{2}}{4} .
\end{aligned}
$$

If $Q=0$, then $\mathcal{A} \oplus Q=0 \mathcal{A}$ is the semi-direct product algebra. Thus $\mathcal{A} \bowtie_{q} \mathcal{A}$ is isomorphic to $\mathcal{A} \ltimes \mathcal{A}$ modulus $q^{2}$.

Now the claim says that $\mathcal{A} \bowtie_{q} \mathcal{A}$ is the result of twisting of $\mathcal{A} \oplus Q \mathcal{A}$ by $q / 2$. Let $(R(\mathcal{A}), \mathcal{A})$ be the graph of $R$. One can easily verify that if $R$ is a $q$-RotaBaxter operator, then $\mathcal{A} \bowtie_{q} \mathcal{A}=\mathcal{A} \bowtie(R(\mathcal{A}), \mathcal{A})$ is a second twilled algebra decomposition. By twisting, we have a twilled algebra $\mathcal{A} \bowtie\left(R(\mathcal{A})+\frac{q}{2} \mathcal{A}, \mathcal{A}\right)$ and

$$
\mathcal{A} \bowtie(R(\mathcal{A}), \mathcal{A})=\mathcal{A} \bowtie_{q} \mathcal{A} \stackrel{e^{\widehat{q / 2}}}{\longrightarrow} \mathcal{A} \oplus_{q^{2} / 4} \mathcal{A}=\mathcal{A} \bowtie\left(R(\mathcal{A})+\frac{q}{2} \mathcal{A}, \mathcal{A}\right) .
$$

Example 5.13 (Rota-Baxter operator $\bmod q^{2}[8]$ ). Let $(\mathcal{A}, R)$ be a Rota-Baxter algebra. We define a linear map $B: \mathcal{A} \rightarrow \mathcal{A}$ by $B(\mathcal{A}):=R(\mathcal{A})+\frac{q}{2} \mathcal{A}$. Then the graph $(B(\mathcal{A}), \mathcal{A})$ of $B$ is a subalgebra of the quasi-twilled algebra $\mathcal{A} \oplus_{q^{2} / 4} \mathcal{A}$. This implies that $B$ is a solution of

$$
B(x) B(y)-B(B(x) y+x B(y))=-\frac{q^{2}}{4} x y .
$$

The right-hand term $\left(q^{2} / 4\right) x y:=\phi_{2}(x, y)$ can be seen as the cocycle-term in (35).

\section{Application}

In this section we give a construction of an associative Nijenhuis operator. First we recall basic properties of the Nijenhuis operator. A linear operator $N: \mathcal{A} \rightarrow \mathcal{A}$ is called associative Nijenhuis operator if $N$ is a solution of

$$
N(x) N(y)=N(N(x) y+x N(y))-N^{2}(x y) .
$$

In general, given a Nijenhuis operator, $x \times_{N} y:=N(x) y+x N(y)-N(x y)$ is a second associative multiplication and it is compatible with the original multiplication. Namely, $x y+t x \times_{N} y$ is a one-parameter family of associative multiplications for any $t \in \mathbb{K}([5])$.

In the following, we assume that $\mathscr{A}$ is an associative algebra, $M$ is an $\mathcal{A}$-bimodule and we denote the multiplication of $\mathcal{A}$ by $*_{\mathcal{A}}$.

Let $\pi: M \rightarrow \mathcal{A}$ be a generalized Rota-Baxter operator, i.e., $\pi$ satisfies the identity

$$
\pi(m) *_{\mathcal{A}} \pi(n)=\pi(\pi(m) \cdot n+m \cdot \pi(n)) .
$$


Here - is the bimodule action of $\mathcal{A}$ on $M$ and $m, n \in M$. We recall the twilled algebra $\mathcal{A} \bowtie M_{\pi}$ in Section 5.1.3. The associative multiplication of $\mathcal{A} \bowtie M_{\pi}$ has the form

$$
(a, m) *(b, n)=\left(a *_{\AA} b+a \cdot_{\pi} n+m \cdot_{\pi} b, a \cdot n+m \cdot b+m \times_{\pi} n\right),
$$

where $\cdot_{\pi}$ means the bimodule action of $M_{\pi}$ on $\mathcal{A}$, explicitly,

$$
\begin{aligned}
m \cdot_{\pi} b & :=\pi(m) *_{\mathcal{A}} b-\pi(m \cdot b), \\
a \cdot_{\pi} n & :=a *_{\mathcal{A}} \pi(n)-\pi(a \cdot n),
\end{aligned}
$$

and $m \times_{\pi} n$ is the associative multiplication of $M_{\pi}$, explicitly,

$$
m \times_{\pi} n:=\pi(m) \cdot n+m \cdot \pi(n) .
$$

We have $\pi\left(m \times_{\pi} n\right)=\pi(m) *_{\mathcal{A}} \pi(n)$.

Consider a linear map $\Omega: \mathcal{A} \rightarrow M_{\pi}$. The map $\Omega$ is a strong Maurer-Cartan operator on a twilled algebra $M_{\pi} \bowtie \mathcal{A}$ if and only if

$$
\begin{aligned}
\Omega\left(a *_{\mathcal{A}} b\right) & =a \cdot \Omega(b)+\Omega(a) \cdot b, \\
\Omega(a) \times_{\pi} \Omega(b) & =\Omega\left(\Omega(a) \cdot_{\pi} b+a \cdot_{\pi} \Omega(b)\right),
\end{aligned}
$$

or, equivalently, $\Omega$ is a solution of

$$
d_{\hat{\mu}} \widehat{\Omega}=\frac{1}{2}[\widehat{\Omega}, \hat{\Omega}]_{\{\hat{\mu}, \hat{\pi}\}}=0 .
$$

We give the main result of this section.

Proposition 6.1. Let $\Omega: \mathcal{A} \rightarrow M_{\pi}$ be a strong Maurer-Cartan operator.

(1) Then a composition map $N:=\pi \Omega$ is an associative Nijenhuis operator on A; namely, $N$ satisfies the condition

$$
N(a) *_{\mathcal{A}} N(b)=N\left(N(a) *_{\mathcal{A}} b+a *_{\mathcal{A}} N(b)\right)-N N\left(a *_{\mathcal{A}} b\right)
$$

for any $a, b \in \mathcal{A}$.

The pair of $(\pi, N)$ is compatible in the following sense.

(2) The composition $N \pi: M \rightarrow \mathcal{A}$ is a second generalized Rota-Baxter operator.

(3) The operators $\pi$ and $N \pi$ are compatible, i.e.,

$$
[\hat{\pi}, \widehat{N \pi}]_{\hat{\mu}}=0 .
$$

This implies that $N \pi$ is strong as the Maurer-Cartan operator and $\pi+t N \pi t \in \mathbb{K}$ is a one-parameter family of generalized Rota-Baxter operators.

Proof. (1) Applying $\pi$ to (38), we have

$$
\pi \Omega(a) *_{\mathcal{A}} \pi \Omega(b)=\pi \Omega\left(\Omega(a) \cdot_{\pi} b+a \cdot_{\pi} \Omega(b)\right) .
$$


On the right-hand side, we get

$\Omega(a) \cdot_{\pi} b+a \cdot_{\pi} \Omega(b)=\pi \Omega(a) *_{\mathcal{A}} b-\pi(\Omega(a) \cdot b)+a *_{\mathcal{A}} \pi \Omega(b)-\pi(a \cdot \Omega(b))$.

By (37), we have

$$
\Omega(a) \cdot_{\pi} b+a \cdot_{\pi} \Omega(b)=\pi \Omega(a) *_{\mathcal{A}} b+a *_{\mathcal{A}} \pi \Omega(b)-\pi \Omega\left(a *_{\mathcal{A}} b\right) .
$$

Thus we obtain the desired condition

$$
\pi \Omega(a) *_{\mathcal{A}} \pi \Omega(b)=\pi \Omega\left(\pi \Omega(a) *_{\mathcal{A}} b+a *_{\mathcal{A}} \pi \Omega(b)\right)-\pi \Omega \pi \Omega\left(a *_{\mathcal{A}} b\right) .
$$

(2) We put $a:=\pi(m)$ and $b:=\pi(n)$ for any $m, n \in M$. Then, by the Nijenhuis condition of $\pi \Omega$, we have

$$
\begin{aligned}
& \pi \Omega \pi(m) *_{\mathcal{A}} \pi \Omega \pi(n) \\
& \quad=\pi \Omega\left(\pi \Omega \pi(m) *_{\mathcal{A}} \pi(n)+\pi(m) *_{\mathcal{A}} \pi \Omega \pi(n)\right)-\pi \Omega \pi \Omega\left(\pi(m) *_{\mathcal{A}} \pi(n)\right) .
\end{aligned}
$$

From the identity (36), we have

$$
\begin{aligned}
& \pi \Omega \pi(m) *_{\mathcal{A}} \pi(n)=\pi(\pi \Omega \pi(m) \cdot n+\Omega \pi(m) \cdot \pi(n)), \\
& \pi(m) *_{\mathcal{A}} \pi \Omega \pi(n)=\pi(\pi(m) \cdot \Omega \pi(n)+m \cdot \pi \Omega \pi(n)),
\end{aligned}
$$

and from the derivation rule, we have

$$
\pi \Omega \pi \Omega\left(\pi(m) *_{\mathcal{A}} \pi(n)\right)=\pi \Omega \pi(\Omega \pi(m) \cdot \pi(n)+\pi(m) \cdot \Omega \pi(n)) .
$$

Thus (39) has the form

$$
\begin{aligned}
& \pi \Omega \pi(m) *_{\mathcal{A}} \pi \Omega \pi(n) \\
& =\pi \Omega \pi(\pi \Omega \pi(m) \cdot n+\Omega \pi(m) \cdot \pi(n)+\pi(m) \cdot \Omega \pi(n)+m \cdot \pi \Omega \pi(n)) \\
& \quad-\pi \Omega \pi(\Omega \pi(m) \cdot \pi(n)+\pi(m) \cdot \Omega \pi(n)) \\
& =\pi \Omega \pi(\pi \Omega \pi(m) \cdot n+m \cdot \pi \Omega \pi(n)),
\end{aligned}
$$

as required.

(3) It is obvious that $\widehat{\pi \Omega \pi}=\hat{\pi} \hat{\Omega} \hat{\pi}$. We have

$$
\begin{aligned}
{[\hat{\pi}, \widehat{\pi \Omega \pi}]_{\hat{\mu}}=} & \{\{\hat{\mu}, \hat{\pi}\}, \hat{\pi} \hat{\Omega} \hat{\pi}\} \\
= & \{\hat{\mu}(\pi \otimes 1)+\hat{\mu}(1 \otimes \hat{\pi})-\hat{\pi} \hat{\mu}, \hat{\pi} \hat{\Omega} \hat{\pi}\} \\
= & \hat{\mu}(\hat{\pi} \otimes \hat{\pi} \hat{\Omega} \hat{\pi})-\hat{\pi} \hat{\Omega} \hat{\pi} \hat{\mu}(\hat{\pi} \otimes 1)+\hat{\mu}(\hat{\pi} \hat{\Omega} \pi \otimes \hat{\pi}) \\
& -\hat{\pi} \hat{\Omega} \hat{\pi} \hat{\mu}(1 \otimes \hat{\pi})-\hat{\pi} \hat{\mu}(\hat{\pi} \hat{\Omega} \hat{\pi} \otimes 1)-\hat{\pi} \hat{\mu}(1 \otimes \hat{\pi} \hat{\Omega} \hat{\pi}),
\end{aligned}
$$


where $\hat{\pi} \hat{\pi}=0$ is used. From the generalized Rota-Baxter condition $[\hat{\pi}, \hat{\pi}]_{\hat{\mu}} / 2=$ $\hat{\mu}(\hat{\pi} \otimes \hat{\pi})-\hat{\pi} \hat{\mu}(\hat{\pi} \otimes 1)-\hat{\pi} \hat{\mu}(1 \otimes \hat{\pi})=0$ we have

$$
\begin{aligned}
(40)= & \hat{\mu}(\hat{\pi} \otimes \hat{\pi} \hat{\Omega} \hat{\pi})-\hat{\pi} \hat{\Omega} \hat{\mu}(\hat{\pi} \otimes \hat{\pi})+\hat{\mu}(\hat{\pi} \hat{\Omega} \pi \otimes \hat{\pi}) \\
& -\hat{\pi} \hat{\mu}(\hat{\pi} \hat{\Omega} \hat{\pi} \otimes 1)-\hat{\pi} \hat{\mu}(1 \otimes \hat{\pi} \hat{\Omega} \hat{\pi}) \\
= & -\hat{\pi} \hat{\Omega} \hat{\mu}(\hat{\pi} \otimes \hat{\pi})+\hat{\mu}(\hat{\pi} \hat{\Omega} \pi \otimes \hat{\pi})-\hat{\pi} \hat{\mu}(\hat{\pi} \hat{\Omega} \hat{\pi} \otimes 1)+\hat{\pi} \hat{\mu}(\hat{\pi} \otimes \hat{\Omega} \hat{\pi}) \\
= & -\hat{\pi} \hat{\Omega} \hat{\mu}(\hat{\pi} \otimes \hat{\pi})+\hat{\pi} \hat{\mu}(\hat{\Omega} \hat{\pi} \otimes \hat{\pi})+\hat{\pi} \hat{\mu}(\hat{\pi} \otimes \hat{\Omega} \hat{\pi}) .
\end{aligned}
$$

Since $\widehat{\Omega}$ is a derivation with respect to $\hat{\mu}$, the last equation of (41) is zero.

Example 6.2. Put $\mathcal{A}:=C^{1}([0,1])$ and $M:=C^{0}([0,1])$. We assume a canonical bimodule action of $\mathcal{A}$ on $M$. An integral operator is a Rota-Baxter operator with weight zero:

$$
\pi: M \rightarrow \mathcal{A}, \quad \pi(f)(x):=\int_{0}^{x} d t f(t) .
$$

Then a derivation

$$
\Omega(f)(x):=\omega(x) \frac{d f}{d x}(x)=\omega(x) f^{\prime}(x), \quad \omega(x) \in C^{0}([0,1]),
$$

from $\mathcal{A}$ to $M_{\pi}$ is a strong Maurer-Cartan operator. The induced Nijenhuis operator on $\mathcal{A}$ is

$$
N(f)(x)=\int_{0}^{x} \omega(t) f^{\prime}(t) d t .
$$

Proof. We only check condition (38). For any $f, g \in \mathcal{A}$,

$$
\Omega(f) \cdot_{\pi} g=\pi \Omega(f) g-\pi(\Omega(f) g)=\int_{0}^{x} d t \omega(t) f^{\prime}(t) g(x)-\int_{0}^{x} d t \omega(t) f^{\prime}(t) g(t) .
$$

We have

$$
\begin{aligned}
& \Omega\left(\Omega(f) \cdot_{\pi} g\right)=\int_{0}^{x} d t \omega(t) f^{\prime}(t) \omega(x) g^{\prime}(x), \\
& \Omega\left(f \cdot_{\pi} \Omega(g)\right)=\omega(x) f^{\prime}(x) \int_{0}^{x} d t \omega(t) g^{\prime}(t) .
\end{aligned}
$$

On the other hand,

$$
\begin{aligned}
\Omega(f) \times_{\pi} \Omega(g) & =\omega(x) f^{\prime}(x) \times_{\pi} \omega(x) g^{\prime}(x) \\
& =\int_{0}^{x} d t \omega(t) f^{\prime}(t) \omega(x) g^{\prime}(x)+\omega(x) f^{\prime}(x) \int_{0}^{x} d t \omega(t) g^{\prime}(t) .
\end{aligned}
$$

Thus we obtain the desired condition. 
We consider two examples in noncommutative cases. In the proof of Example 6.2, we used the commutativity of only $\omega$. Hence if $\omega$ is 1 or a central element, then a similar proof holds in the noncommutative setting.

Example 6.3. Let $\mathcal{A}$ be an associative algebra and let $\mathcal{A}[[v]]$ be an algebra of formal series with coefficients in $\mathcal{A}$. The multiplication on $\mathcal{A}[[v]]$ is defined by

$$
a_{i} v^{i} * b_{j} v^{j}:=a_{i} b_{j} v^{i+j}, \quad a_{i}, b_{j} \in \mathcal{A},
$$

where $\sum$ is omitted. We define a formal integral operator

$$
\int d v a_{i} v^{i}:=\frac{1}{i+1} a_{i} v^{i+1}, \quad a_{i} \in \mathcal{A} .
$$

The integral operator is a Rota-Baxter operator with weight zero. The formal derivation operator is a strong Maurer-Cartan operator

$$
\Omega\left(a_{i} v^{i}\right):=z_{k} v^{k} \frac{d}{d v}\left(a_{i} v^{i}\right):=i z_{k} a_{i} v^{i+k-1}, \quad z_{k} \in Z(\mathcal{A}) .
$$

Here $Z(\mathcal{A})$ is the space of central elements. The induced Nijenhuis operator is

$$
N\left(a_{i} v^{i}\right):=\frac{i}{i+k} z_{k} a_{i} v^{i+k} .
$$

Example 6.4. Let $W\left\langle x, \partial_{x}\right\rangle$ be the Weyl algebra. Define a formal integral operator for the normal basis of the Weyl algebra by

$$
\int d x \partial_{x}^{i} * x^{j}:=\frac{1}{1+j} \partial_{x}^{i} * x^{j+1}, \quad i, j \geq 0 .
$$

Then this integral operator is a Rota-Baxter operator with weight zero (see [23]). Put $\Omega:=i_{\partial_{x}}$. Then $\Omega$ is a strong Maurer-Cartan operator. Thus the composition map

$$
N(u):=\int d x \Omega(u)=\int d x\left[\partial_{x}, u\right]
$$

is a Nijenhuis operator on $W\left\langle x, \partial_{x}\right\rangle$. Since an arbitrary element $u$ has the form $u:=k_{i j} \partial_{x}^{i} * x^{j(j \neq 0)}+k_{i} \partial_{x}^{i}+k$, we have $N(u)=k_{i j} \partial_{x}^{i} * x^{j(j \neq 0)}$. Thus $N$ is a projection onto the space of elements of the form $k_{i j} \partial_{x}^{i} * x^{j(j \neq 0)}$.

\section{References}

[1] M. Aguiar, Pre-Poisson algebras. Lett. Math. Phys. 54 (2000), 263-277. Zbl 1032.17038 MR 1846958 
[2] M. Aguiar, Infinitesimal Hopf algebras. In New trends in Hopf algebra theory (La Falda, 1999), Contemp. Math. 267, Amer. Math. Soc., Providence, RI, 2000, 1-29. Zbl 0982.16028 MR 1800704

[3] M. Aguiar, On the associative analog of Lie bialgebras. J. Algebra 244 (2001), 492-532. Zbl 0991.16033 MR 1859038

[4] G. Baxter, An analytic problem whose solution follows from a simple algebraic identity. Pacific J. Math. 10 (1960), 731-742. Zbl 0095.12705 MR 0119224

[5] J. F. Cariñena, J. Grabowski, and G. Marmo, Quantum bi-Hamiltonian systems. Internat. J. Modern Phys. A 15 (2000), 4797-4810. Zbl 1002.81026 MR 1800909

[6] M. Doubek, M. Markl, and P. Zima, Deformation theory (lecture notes). Arch. Math. (Brno) 43 (2007), 333-371. Zbl 05596016 MR 2381782

[7] V. G. Drinfeld, Quasi-Hopf algebras. Algebra i Analiz 1 (1989), 114-148; English transl. Leningr. Math. J. 1 (1990), 1419-1457. Zbl 0718.16033 MR 1047964

[8] K. Ebrahimi-Fard, Loday-type algebras and the Rota-Baxter relation. Lett. Math. Phys. 61 (2002), 139-147. Zbl 1035.17001 MR 1936573

[9] S. A. Joni and G.-C. Rota, Coalgebras and bialgebras in combinatorics. Stud. Appl. Math. 61 (1979), 93-139. Zbl 0471.05020 MR 544721

[10] Y. Kosmann-Schwarzbach, Jacobian quasi-bialgebras and quasi-Poisson Lie groups. In Mathematical aspects of classical field theory (Seattle, WA, 1991), Contemp. Math. 132, Amer. Math. Soc., Providence, RI, 1992, 459-489. Zbl 0847.17020 MR 1188453

[11] Y. Kosmann-Schwarzbach, From Poisson algebras to Gerstenhaber algebras. Ann. Inst. Fourier (Grenoble) 46 (1996), 1243-1274. Zbl 0858.17027 MR 1427124

[12] Y. Kosmann-Schwarzbach, Quasi, twisted, and all that ... in Poisson geometry and Lie algebroid theory. In The breadth of symplectic and Poisson geometry, Progr. Math. 232, Birkhäuser, Boston 2005, 363-389. Zbl 1079.53126 MR 2103012

[13] Y. Kosmann-Schwarzbach and F. Magri, Poisson-Lie groups and complete integrability. I. Drinfeld bialgebras, dual extensions and their canonical representations. Ann. Inst. H. Poincaré Phys. Théor. 49 (1988), 433-460. Zbl 0667.16005 MR 988946

[14] Y. Kosmann-Schwarzbach and F. Magri, Poisson-Nijenhuis structures. Ann. Inst. H. Poincaré Phys. Théor. 53 (1990), 35-81. Zbl 0707.58048 MR 1077465

[15] T. Lada and M. Markl, Strongly homotopy Lie algebras. Comm. Algebra 23 (1995), 2147-2161. Zbl 0999.17019 MR 1327129

[16] Z.-J. Liu, A. Weinstein, and P. Xu, Manin triples for Lie bialgebroids. J. Differential Geom. 45 (1997), 547-574. Zbl 0885.58030 MR 1472888

[17] J.-H. Lu and A. Weinstein, Poisson Lie groups, dressing transformations, and Bruhat decompositions. J. Differential Geom. 31 (1990), 501-526. Zbl 0673.58018 MR 1037412

[18] G.-C. Rota, Baxter algebras and combinatorial identities. I, II. Bull. Amer. Math. Soc. 75 (1969), 325-329; ibid. 75 (1969), 330-334. Zbl 0192.33801 Zbl 0319.05008 MR 0244070

[19] G.-C. Rota, Baxter operators, an introduction. In Gian-Carlo Rota on combinatorics, Contemp. Math., Birkhäuser, Boston 1995, 504-512. 
[20] G.-C. Rota, Gian-Carlo Rota on analysis and probability. Contemp. Math., Birkhäuser, Boston 2003. Zbl 1159.01014 MR 1944526

[21] D. Roytenberg, Quasi-Lie bialgebroids and twisted Poisson manifolds. Lett. Math. Phys. 61 (2002), 123-137. Zbl 1027.53104 MR 1936572

[22] D. Roytenberg, On the structure of graded symplectic supermanifolds and Courant algebroids. In Quantization, Poisson brackets and beyond (Manchester, 2001), Contemp. Math. 315, Amer. Math. Soc., Providence, RI, 2002, 169-185. Zbl 1036.53057 MR 1958835

[23] K. Uchino, Quantum analogy of Poisson geometry, related dendriform algebras and RotaBaxter operators. Lett. Math. Phys. 85 (2008), 91-109. Zbl 05544981 MR 2443932

[24] K. Uchino, Derived brackets and sh Leibniz algebras. Preprint 2009. arXiv:0902.0044

[25] I. Vaisman, Complementary 2-forms of Poisson structures. Compositio Math. 101 (1996), 55-75. Zbl 0853.58056 MR 1390832

Received December 10, 2007; revised February 8, 2009

K. Uchino, Tokyo University of Science, Wakamiya-cho 26, Shinjuku-ku, Tokyo-to, 162-0827, Japan

E-mail: k_uchino@oct.rikadai.jp 